This item was submitted to Loughborough's Research Repository by the author.

Items in Figshare are protected by copyright, with all rights reserved, unless otherwise indicated.

\title{
A framework for design and optimization of tapered composite structures. Part I: from individual panel to global blending structure
}

PLEASE CITE THE PUBLISHED VERSION

http://dx.doi.org/10.1016/j.compstruct.2016.05.095

\section{PUBLISHER}

(C) Elsevier Ltd.

VERSION

AM (Accepted Manuscript)

\section{PUBLISHER STATEMENT}

This work is made available according to the conditions of the Creative Commons Attribution-NonCommercialNoDerivatives 4.0 International (CC BY-NC-ND 4.0) licence. Full details of this licence are available at: https://creativecommons.org/licenses/by-nc-nd/4.0/

\section{LICENCE}

CC BY-NC-ND 4.0

\section{REPOSITORY RECORD}

Jing, Zhao, Qin Sun, and Vadim V. Silberschmidt. 2019. "A Framework for Design and Optimization of Tapered Composite Structures. Part I: From Individual Panel to Global Blending Structure". figshare. https://hdl.handle.net/2134/23024. 


\title{
A framework for design and optimization of tapered composite structures. Part I: From individual panel to global blending structure
}

\author{
Zhao Jing a,b, Qin Sun ${ }^{\mathrm{a}, *}$, Vadim V. Silberschmidt ${ }^{\mathrm{b}}$ \\ a School of Aeronautics, Northwestern Polytechnical University, Xi'an 710072, China \\ ${ }^{\mathrm{b}}$ Wolfson School of Mechanical and Manufacturing Engineering, Loughborough University, LE11 3TU Leicestershire, UK
}

Keywords:

Tapered composite structures

Structural design optimization

Large-scale

Stacking sequence

Buckling

Manufacturing constraints

\begin{abstract}
A B S T R A C T
A novel framework to design tapered composite structures is proposed for buckling analysis with multiple manufacturing constraints, with variables dealt with separately at different levels and manufacturing constraints divided and imposed in each step. The framework is based on two techniques - a sequential permutation table (SPT) method and a global shared-layer blending (GSLB) method; a design concept is extending from an individual panel to the overall structure by improving its blending property. A problem to design an 18-panel tapered composite structure is adopted to study and validate the proposed framework; a detailed design process is executed step by step to demonstrate the improvements of blending property. Multiple manufacturing constraints are analyzed for the obtained optimal solution, which is also compared with previous studies. The high efficiency of the proposed framework implies its potential for design of large-scale composite structures with complex manufacturing constraints.
\end{abstract}

\section{Introduction}

Composite materials are widely employed in industries for their excellent mechanical properties: high values of strength-to-weight and stiffness-to-weight ratios. Weight reduction provides a stronger motivation for the use of composites in aerospace and transportation applications in particular. The way to save weight of composites components and structures in engineering is to tailor layers continuously through the structure to change their properties (e.g., stiffness, strength, etc.), controlled by a designer. As a result, variable thickness can be tailored spatially under varying loads, resulting in ply drops in the structure that cause stress concentration and increase a risk of delamination etc. The design of tapered composite structures has received much attention from researchers for its potential in tailoring properties and saving weight $[1,27,28]$.

A high freedom of design space allows designs of laminated structures according to applied loads and manufacturing requirements. The variable thickness, ply orientation, layer shape and stacking sequence not only increase design flexibility, but also bring great challenges for structural designers [2]. Because of high-dimensional design space and complicated constraints,

\footnotetext{
* Corresponding author.

E-mail address: sunqin@nwpu.edu.cn (Q. Sun).
}

heuristic algorithms were adopted to attack this problem [3]. The most widely used is a genetic algorithm (GA), an 18-panel horseshoes design problem was first proposed in [4], with a commercial software DARWIN used for optimization. After that, many researchers used improved GA [5,6] and modified GA [7-11] to solve this problem. The main concern of these studies is the efficiency of the algorithm and a blending requirement. Hence, specific techniques were developed to address the blending problem, such as: guide-based design [6], inward and outward blending [6,8,10], generalized blending [7], a ply drop sequence (PDS) [9], a blending constraint can be imposed by modifying a chromosome implemented in the GA [11] to improve efficiency. Further, the GA was also applied to a wing-box design problem [12-14]. Particularly, a shared layer blending (SLB) [14] method was first introduced to solve a blending problem. In recent years, various methods were used by researchers in blending design. One of the outstanding work was presented in [15], a stacking sequence table (SST) based evolutionary algorithm (EA) is developed for the 18-panel blended design [4] with multiple manufacturing constraints. A backtracking method is popular to ensure the blending and manufacturing rules [16-18]. Besides, an ant-colony (ACO) system was applied for blended bistable laminates for morphing applications [19]. Additionally, the multi-phase topology optimization [20,21] based methods - Discrete Material Optimization (DMO) and Shape Functions with Penalization (SFP) - are widely used for topology optimization and stacking sequence design of composite structures. 
Apart from the above methods, a three-phase design optimization process was introduced in [22], leading from concept design to final ply-book details design. Ply tailoring, numbers of plies and a stacking sequence are designed separately in three phases with manufacturing constraints divided and imposed in each phase. Most recently, Jing [23-25] developed algorithms based on a classical lamination theory (CLT) to solve stacking-sequence optimization problem. A permutation search (PS) algorithm [23] was developed for sequence optimization as well as a sequential permutation table (SPT) method [25], with the buckling load factor expressed as a linear function of a sum of buckling load factors at every stacking position corresponding to ply orientation. Besides, a global shared-layer blending (GSLB) method [24] was proposed based on the SLB method [14] to identify layer shape/ maintain blending property according to the structural thickness distribution. From the above analysis, blending is the main concern of design problem, because a higher blending property/fewer plydrops can decrease stress concentration as well as improve structural integrity. Moreover, the design of composite structures with complicated manufacturing constraints is more difficult [15].

The main reason for researchers to apply various heuristic algorithms is the high-dimensional discrete variables, in which the form of these variables is different and they coupled with each other in a design process. For instance, the thickness varying may cause ply shape variation; the stacking sequence is influenced by ply orientations when the thickness is fixed. Thus, it is hard to divide different forms of variables in a design process or deal with them separately. However, heuristic algorithms are sensitive to predefined parameters; they search optimum from an initial point/multi-points, making it easy to reach a local optimum, especially when the variable space is divided by constraints. For a largescale optimization problem, heuristic algorithms may have a very low convergence rate or unable to find the global optimum. The difficulties of previous studies and methods motivated this work. An entire design framework for design and optimization of tapered composite structures is proposed, with variables divided at different levels to deal with, while manufacturing constraints are divided and imposed for different type of variables.

The remainder of this article is organized as follows: in Section 2, the optimization model is formulated, then the problem is analyzed and decomposed, followed by the introduction of the design framework. Detailed techniques for the design framework are presented in Section 3. A benchmark problem is adopted to verify the proposed framework in Section 4. Finally, conclusions and suggestions are given in Section 5.

\section{Design framework}

\subsection{Design modeling}

According to the classical lamination theory $[26,30]$ :

$$
\left[\begin{array}{c}
F \\
M
\end{array}\right]=\left[\begin{array}{ll}
A & B \\
B & D
\end{array}\right]\left[\begin{array}{l}
\varepsilon^{0} \\
k
\end{array}\right],
$$

where $F$ is the vector of in-plane loads; $M$ is the vector of out-ofplane moments; $\varepsilon^{0}$ is the vector of mid-plane strains, and $\kappa$ is the vector of plate curvatures. The explicit formulation of Eq. (1) is:

$$
\left[\begin{array}{c}
F_{x} \\
F_{y} \\
F_{x y} \\
M_{x} \\
M_{y} \\
M_{x y}
\end{array}\right]=\left[\begin{array}{llllll}
A_{11} & A_{12} & A_{16} & B_{11} & B_{12} & B_{16} \\
A_{21} & A_{22} & A_{26} & B_{21} & B_{22} & B_{26} \\
A_{16} & A_{26} & A_{66} & B_{16} & B_{26} & B_{66} \\
B_{11} & B_{12} & B_{16} & D_{11} & D_{12} & D_{16} \\
B_{21} & B_{22} & B_{26} & D_{21} & D_{22} & D_{26} \\
B_{16} & B_{26} & B_{66} & D_{16} & D_{26} & D_{66}
\end{array}\right]\left[\begin{array}{c}
\varepsilon_{x}^{0} \\
\varepsilon_{y}^{0} \\
\varepsilon_{x y}^{0} \\
\kappa_{x} \\
\kappa_{y} \\
\kappa_{x y}
\end{array}\right],
$$

where

$$
\begin{aligned}
& A_{i j}=\int_{-h / 2}^{h / 2}\left(\bar{Q}_{i j}\right)_{k} d z=\sum_{k=1}^{N}\left(\bar{Q}_{i j}\right)_{k}\left(z_{k}-z_{k-1}\right) \\
& B_{i j}=\int_{-h / 2}^{h / 2}\left(\bar{Q}_{i j}\right)_{k} z d z=\frac{1}{2} \sum_{k=1}^{N}\left(\bar{Q}_{i j}\right)_{k}\left(z_{k}^{2}-z_{k-1}^{2}\right), \\
& D_{i j}=\int_{-h / 2}^{h / 2}\left(\bar{Q}_{i j}\right)_{k} z^{2} d z=\frac{1}{3} \sum_{k=1}^{N}\left(\bar{Q}_{i j}\right)_{k}\left(z_{k}^{3}-z_{k-1}^{3}\right)
\end{aligned}
$$

where $A_{i j}$ are extensional stiffness, $B_{i j}$ are bending-extension coupling stiffness, and $D_{i j}$ are bending stiffness, $\left(\bar{Q}_{i j}\right)_{k}$ are transformed reduced stiffness. The definition of $z_{k}$ is based on a number of layers in the laminate from the bottom to the top (see Fig. 1(a) and (b)) and $z_{k}=t(k-N / 2), N$ is the total number of plies in a laminate. For a symmetrical laminate, the integration of Eq. (3) can be limited to its half (see Fig. 1(c) and (d)) and multiply $2 ; z_{k}$ is different for even and odd ply numbers of the laminate. In Fig. 1 (c) $z_{k}=t k$ for even plies, while in Fig. 1(d) $z_{k}=t(k-1 / 2)$ for odd plies since the mid-plane of the laminate coincides with the first ply's mid-plane. As a result, Eq. (3) is changed for Fig. 1(c) and (d):

$N$ is an even number : $z_{k}=t k$

$$
\begin{aligned}
& A_{i j}=2 \sum_{k=1}^{N / 2}\left(\bar{Q}_{i j}\right)_{k}\left(z_{k}-z_{k-1}\right)=2 t \sum_{k=1}^{N / 2}\left(\bar{Q}_{i j}\right)_{k}, \\
& B_{i j}=\sum_{k=1}^{N / 2}\left(\bar{Q}_{i j}\right)_{k}\left(z_{k}^{2}-z_{k-1}^{2}\right)=2 t^{2} \sum_{k=1}^{N / 2}\left(\bar{Q}_{i j}\right)_{k}\left(k-\frac{1}{2}\right), \\
& D_{i j}=\frac{2}{3} \sum_{k=1}^{N / 2}\left(\bar{Q}_{i j}\right)_{k}\left(z_{k}^{3}-z_{k-1}^{3}\right)=2 t^{3} \sum_{k=1}^{N / 2}\left(\bar{Q}_{i j}\right)_{k}\left(k^{2}-k+\frac{1}{3}\right),
\end{aligned}
$$

$N$ is an odd number : $z_{k}=t(k-1 / 2)$, when $k=1$, there is only

$$
\begin{aligned}
& \text { one lamina } A_{i j}=2 \sum_{k=1}^{(N+1) / 2}\left(\bar{Q}_{i j}\right)_{k}\left(z_{k}-z_{k-1}\right) \\
& =\left\{\begin{array}{l}
t\left(\bar{Q}_{i j}\right)_{1}, N=1 \text { and } k=1 \\
2 t\left[\sum_{k=2}^{(N+1) / 2}\left(\bar{Q}_{i j}\right)_{k}+\frac{1}{2}\left(\bar{Q}_{i j}\right)_{1}\right], N \geqslant 3 \text { and } k \geqslant 2
\end{array}\right.
\end{aligned}
$$

$$
B_{i j}=\sum_{k=1}^{(N+1) / 2}\left(\bar{Q}_{i j}\right)_{k}\left(z_{k}^{2}-z_{k-1}^{2}\right)
$$

$$
=\left\{\begin{array}{l}
\frac{1}{4} t^{2}\left(\bar{Q}_{i j}\right)_{1}, N=1 \text { and } k=1 \\
2 t^{2}\left[\sum_{k=2}^{(N+1) / 2}\left(\bar{Q}_{i j}\right)_{k}(k-1)+\frac{1}{8}\left(\bar{Q}_{i j}\right)_{1}\right], N \geqslant 3 \text { and } k \geqslant 2
\end{array}\right.
$$

$$
\begin{aligned}
D_{i j}= & \frac{2}{3} \sum_{k=1}^{(N+1) / 2}\left(\bar{Q}_{i j}\right)_{k}\left(z_{k}^{3}-z_{k-1}^{3}\right) \\
= & \left\{\begin{array}{l}
\frac{1}{12} t^{3}\left(\bar{Q}_{i j}\right)_{1}, N=1 \text { and } k=1 \\
2 t^{3}\left[\sum_{k=2}^{(N+1) / 2}\left(\bar{Q}_{i j}\right)_{k}\left(k^{2}-2 k+\frac{13}{12}\right)+\frac{1}{24}\left(\bar{Q}_{i j}\right)_{1}\right], N \geqslant 3 \text { and } k \geqslant 2
\end{array}\right.
\end{aligned}
$$




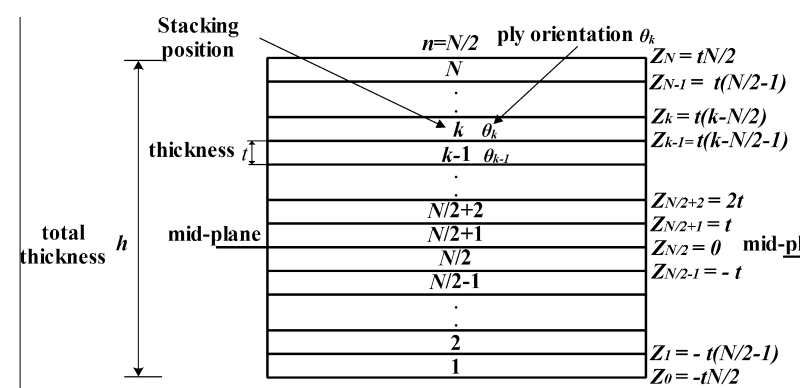

(a) $N$ is an even number, from bottom to top

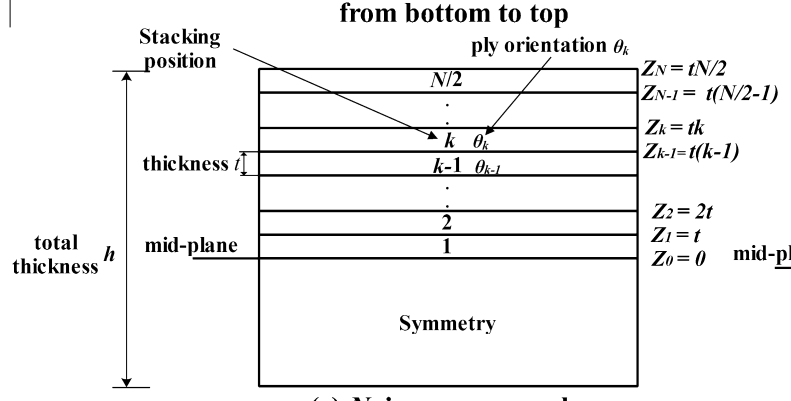

(c) $N$ is an even number, from mid-plane to top

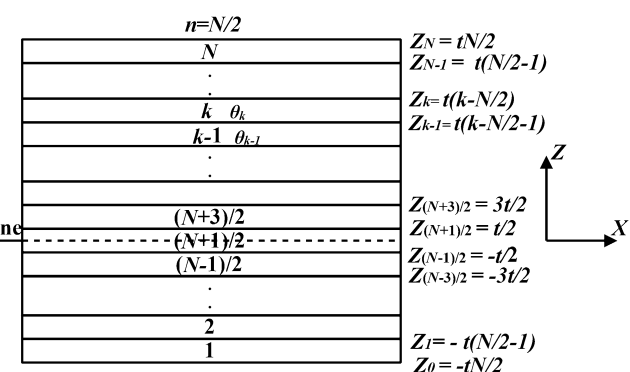

(b) $N$ is an odd number, from bottom to top

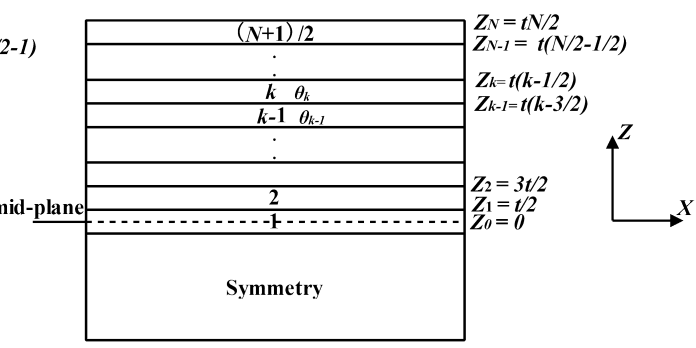

(d) $N$ is an odd number, from mid-plane to top

Fig. 1. Different definitions of $z_{k}$ in a laminate: (a) and (b) are from the bottom to the top; (c) and (d) are from the mid-plane to the top for symmetrical laminate.

Thus, the discrete stiffness parameters for every stacking position $k$ can be obtained:

$N$ is an even number : $k=1,2, \ldots, N / 2$

$$
\begin{aligned}
& \left(A_{i j}\right)_{k}=2 t\left(\bar{Q}_{i j}\right)_{k}, \\
& \left(B_{i j}\right)_{k}=2 t^{2}\left(\bar{Q}_{i j}\right)_{k}\left(k-\frac{1}{2}\right), \\
& \left(D_{i j}\right)_{k}=2 t^{3}\left(\bar{Q}_{i j}\right)_{k}\left(k^{2}-k+\frac{1}{3}\right),
\end{aligned}
$$

$N$ is an odd number :

$$
\begin{aligned}
& \left(A_{i j}\right)_{k}=\left\{\begin{array}{l}
t\left(\bar{Q}_{i j}\right)_{k}, k=1 \\
2 t\left(\bar{Q}_{i j}\right)_{k}, k=2, \ldots,(N+1) / 2
\end{array}\right. \\
& \left(B_{i j}\right)_{k}=\left\{\begin{array}{l}
\frac{1}{4} t^{2}\left(\bar{Q}_{i j}\right)_{1}, k=1 \\
2 t^{2}\left(\bar{Q}_{i j}\right)_{k}(k-1), k=2, \ldots,(N+1) / 2
\end{array}\right. \\
& \left(D_{i j}\right)_{k}=\left\{\begin{array}{l}
\frac{1}{12} t^{3}\left(\bar{Q}_{i j}\right)_{1}, k=1 \\
2 t^{3}\left(\bar{Q}_{i j}\right)_{k}\left(k^{2}-2 k+\frac{13}{12}\right), k=2, \ldots,(N+1) / 2
\end{array}\right.
\end{aligned}
$$

It should be pointed out that in Eq. (5) stiffness parameters $\left(A_{i j}\right)_{k},\left(B_{i j}\right)_{k},\left(D_{i j}\right)_{k}$ at the stacking position $k$ are the sum of stiffness of two layers located symmetrically with regard to the mid-plane. Thus, at every stacking position $k$ the stiffness parameters can be calculated independently. This is convenient for design of a stacking sequence, since bending stiffness $D_{i j}$ are controlled by the stacking sequence when thickness is fixed.

The objective in the design of tapered composite structure is minimizing the weight while satisfying mechanical and manufacturing requirements for engineering applications. A tapered composite structure consists of individual panels with variable thickness, where linear buckling is one of the main critical failure modes. In this work, buckling of symmetrical and balance laminates with even ply numbers, simply supported on boundaries, under normal compressive loads $\left(F_{x}, F_{y}\right)$ is analyzed (see Fig. 2). The formulation of buckling load factor is [25]:

$$
\lambda_{c b}=\pi^{2} \frac{\left[D_{11}\left(\frac{m}{a}\right)^{4}+2\left(D_{12}+2 D_{66}\right)\left(\frac{m}{a}\right)^{2}\left(\frac{n}{b}\right)^{2}+D_{22}\left(\frac{n}{b}\right)^{4}\right]}{F_{x}\left(\frac{m}{a}\right)^{2}+F_{y}\left(\frac{n}{b}\right)^{2}},
$$

where $m$ and $n$ are the numbers of half-waves along the $x$ and $y$ directions respectively; $a$ and $b$ are the length and width of the panel respectively. $D_{11}, D_{12}, D_{22}$ and $D_{66}$ are the bending stiffness of the laminate ( $D_{16}$ and $D_{26}$ are neglected for the orthotropic plate). Since the forms $D_{11}, D_{12}, D_{22}$ and $D_{66}$ can be expressed as discrete forms of $\left(D_{11}\right)_{k},\left(D_{12}\right)_{k},\left(D_{22}\right)_{k}$ and $\left(D_{66}\right)_{k}$, respectively (see Eq. (4) and (5)). The buckling load factor can be expressed as [25]:

$$
\begin{aligned}
\lambda_{c b}= & \sum_{k=1}^{N / 2}\left(\lambda_{c b}\right)_{k}=\frac{\pi^{2}}{F_{x}\left(\frac{m}{a}\right)^{2}+F_{y}\left(\frac{n}{b}\right)^{2}} \\
& \times \sum_{k=1}^{N / 2}\left[\left(D_{11}\right)_{k}\left(\frac{m}{a}\right)^{4}+2\left(\left(D_{12}\right)_{k}+2\left(D_{66}\right)_{k}\right)\left(\frac{m}{a}\right)^{2}\left(\frac{n}{b}\right)^{2}+\left(D_{22}\right)_{k}\left(\frac{n}{b}\right)^{4}\right]
\end{aligned}
$$

where $\lambda_{c b}$ is expressed as a discrete form of a sum of $\left(\lambda_{c b}\right)_{k}$ at stacking position $k$. And $\left(\lambda_{c b}\right)_{k}$ is expressed as a linear sum of $\left(D_{11}\right)_{k}$, $\left(D_{12}\right)_{k},\left(D_{22}\right)_{k}$ and $\left(D_{66}\right)_{k}$. Combining with Eq. (5), the buckling load factor $\lambda_{c b}$ satisfies the superposition principle and, thus, the stacking sequence can be designed linearly with variable ply orientation $\theta_{k}$ at each stacking position $k$. That is to say, the ply orientation $\theta_{k}$ can be selected one by one at each stacking position to maximize the $\left(\lambda_{c b}\right)_{k}$, since ply orientation $\theta_{k}$ is independent variable for a specific stacking position $k$.

On the other hand, multiple manufacturing constraints should be considered in a design process. From the engineering perspective, the ply drops in tapered composite structures should be avoided or as few as possible to decrease stress concentration and improve structural integrity. A detailed design guidelines are introduced in [15]. The objective function for optimization is formulated as follows: 
Min : $\quad W=\sum_{i=1}^{P} \rho t A_{i} N_{i}$

Sub: (1) Constraint for buckling: $\lambda_{c b}^{i} \geqslant 1, i=1,2, \ldots, P$

(2) Constraints for individual panel or each ply drops:

CT1. Symmetry. Stacking sequence should be symmetric about the midplane.

CT2. Balance. The number of ply orientations $+\theta$ and $-\theta\left(\theta \neq 0^{\circ}\right.$ or $\left.90^{\circ}\right)$ should be the same in laminate.

$C T 3$. Contiguity. No more than $U(U \leqslant 3)$ same orientations should be stacked together; hence, no more than $[U / 2]$ layers stacked together at the midplane in half laminate.

CT4. Disorientation. Difference between the orientations of two consecutive plies should not exceed $45^{\circ}$.

CT5. Damtol. No $0^{\circ}$ ply should be placed on the lower and upper surfaces of the laminate.

CT6. Covering. Covering plies on the lower and upper surfaces of the laminate should not be dropped.

CT7. Maximum taper slope. The taper angle should not exceed $7^{\circ}$.

CT8. Max - stopping. No more than four plies should be stopped at the same increment of thickness.

CT9. Internal continuity. A continuous ply should be kept every four consecutive dropped plies.

CT10.Ply - drop alternation. Ply drops should be located alternately close and far from the mid plane.

CT11. Taper guidelines. All laminates in the taper section should fulfil the maximum possible extend of the laminate design guidelines.

(3) Constraints for global structure:

CT12. Continuity. All plies from a thinner panel must cover the whole structure.

CT13. $\Delta n-$ rule. A maximum number of ply drops $\Delta n$ between adjacent panels.

Here, $W$ is the weight of the tapered composite structure, $P$ is the total number of panels, $\rho$ is the density, $t$ is the thickness of an individual ply, $A_{i}$ is the area of panel $i, N_{i}$ (even number) is the total number of plies of panel $i$. $\lambda_{c b}^{i}$ is the buckling load factor of panel $i$. The constraints $C T 1$ to $C T 13$ are divided into three parts: the first part is the buckling constraint; the second part is for an individual panel; the third part is for the whole structure. The difficulty of this design problem is the implementation of complicated constraints. Because constraints are coupled with each other, for instance, the balance constraint requires the same number of ply orientations $+\theta$ and $-\theta$, it affects both contiguity and disorientation constraints since they impose limitation on the proportion of ply orientations. Thus, the constraints should be divided and imposed in multi-steps to be dealt with.

\subsection{Decomposition of design variables}

In Eq. (7), the buckling load factor $\lambda_{c b}$ is the function of bending stiffness $D$, panel size $(a, b)$, half waves $(m, n)$ and compressive loads $\left(F_{x}, F_{y}\right)$. For a given panel with compressive loads, half waves $(m, n)$ are determined [29]; hence, the only variable is bending stiffness $D$. At the same time, Eq. (4) shows that bending stiffness $D$ is a cubic function of the thickness; thus, for a specific position $k$, the only variable is $\left(\bar{Q}_{i j}\right)_{k}$, which is determined by ply orientation

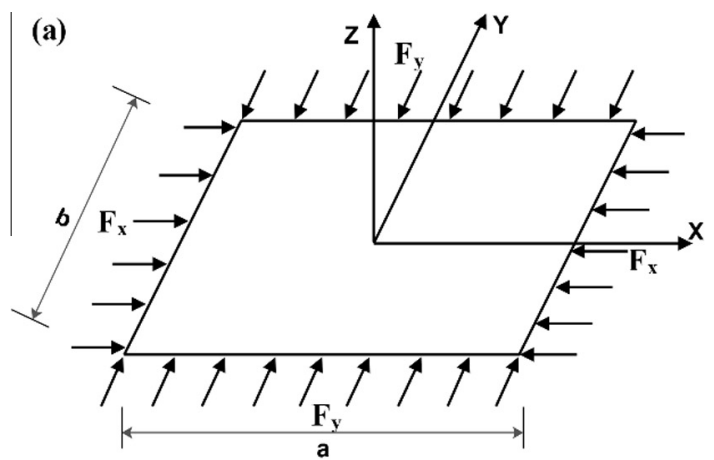

$\theta_{k}$. Since $\left(\bar{Q}_{i j}\right)_{k}$ is a triangle function of $\theta_{k}$, its values are limited by the range of triangle function $[-1,1]$. Thus, for a fixed thickness panel, the design variables are the ply orientations for every stacking position. In contrast, the influence of thickness is larger than the ply orientations for a variable thickness panel (see Eq. (4)).

Optimization of tapered composite structures is more complicated than design of a single panel, since it is composed of multiple panels with different thicknesses, and the thickness are variables. However, the objective is to obtain the minimum weight with corresponding thickness restricted to the manufacturing constraints (see Eq. (8)). As analyzed above, these variables and constraints can be divided since their influences on the buckling load factors are different. Fig. 3 demonstrates the relationship of different variables and their design levels.

In Fig. 3, according to Eq. (7), thickness is the key factor in determination of the buckling load factor; thus, thickness of different panels should be identified at level 1 . The blending requirement is a critical constraint; based on the thickness obtained at level 1, the ply shape should be determined at level 2. The shared layer is treated as one integrated layer, and the corresponding stacking position is decided by its area: the larger the area, the outer the shared layer (the stacking position is assumed to identify the ply orientation, sequence will be optimized in further design). Thus, ply orientation can be selected for shared layers with various

\section{(b)}

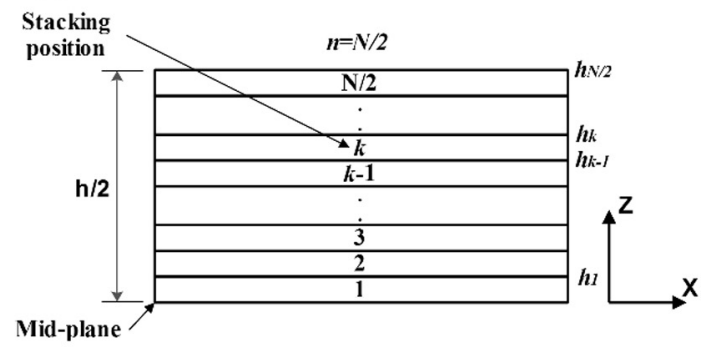

Fig. 2. (a) Loads and geometry of laminate; (b) stacking-sequence definition. 


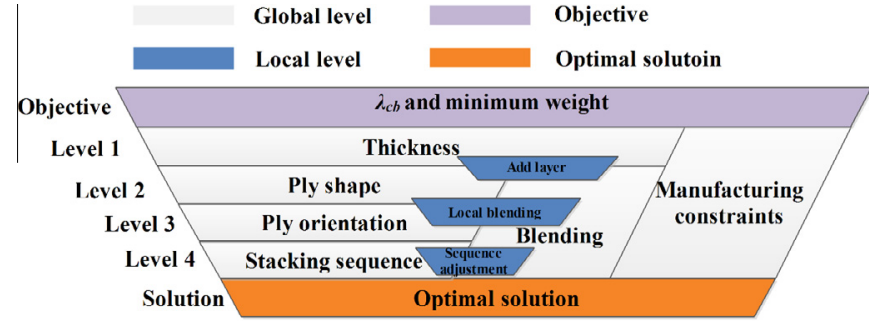

Fig. 3. Variables are divided and their design levels for optimization of tapered composite structures.

shapes at level 3. Finally, the stacking sequence is optimized at level 4. The above four levels are at the global level, considering the coupling of blending, buckling and manufacturing constraints, the solution should be adjusted at a local level. First, layers are added to the panels those violate the buckling constraint: the thickness, the ply shape and the blending constraint are considered simultaneously. Second, ply orientations are adjusted to improve the blending property considering the ply orientation, the ply shape and the blending constraint. Third, the stacking sequence is adjusted to decrease continuity ply drops (the ply shape does not change). Obviously, the manufacturing constraints are enforced at every step in Fig. 3 at the global and local levels. Based on the decomposition of design variables, specific design techniques are developed for different variables in the next section.

\subsection{Design techniques}

A new framework for design of tapered composite structures is proposed based on two techniques in [24,25]; the design concept is extending from an individual panel to the overall structure by improving its blending property. All complex constraints are considered, they are divided into different sets and implemented at various steps. A stacking sequence of individual panel is obtained by using sequential permutation table (SPT) [25] without consider- ing a blending requirement. However, to maintain the maximal blending property, the GSLB method [24] is adopted. Additionally, several new techniques are developed and combined with the previous two techniques.

There are three main stages in the design process: first, obtain the minimum-weight structure without considering constraints, SPT method is used to figure out the minimum-thickness distribution of the structure. Second, blending and sequence design are implemented, with multiple constraints imposed. Third, maximizing the blending property without increasing weight, all constraints are enforced. The detailed steps are introduced as follows. First of all, the SPT method is applied to each panel to obtain contribution values of each ply orientation, these ply orientations are sorted in a table from best to worst according to their contribution values, the table is named multi-panel sequential permutation table (MSPT). Second, the minimum thickness of each panel is calculated based on the MSPT by setting the best ply orientation in the laminate at all stacking positions; thus, the minimum-thickness distribution can be identified. Third, assume that all ply orientations are the same in the minimum-thickness distribution, the GSLB method is adopted to predict the shape of shared layers. Fourth, the ply orientations of the shared layers are identified using a ply-orientation identification technique, and the balance requirement is imposed. Fifth, a sequence optimization technique is used to optimize the sequence of shared layers; here, some local constraints are imposed in individual panel. Sixth, an add layer technique is adopted to add layers to panels violate buckling constraint, where lightweight and blending requirements are considered. After that, the number of ply orientations is changed, the GSLB method and sequence optimization strategy are employed again. Besides, buckling load factors of some panels have big margins, the local blending technique is used to improve the structural integrity by decreasing buckling load factors. Eventually, the GSLB and sequence optimization strategy are implemented again, the sequence-adjustment technique is used to decrease continuity ply drops. The design concept and its flowchart are presented in Fig. 4.

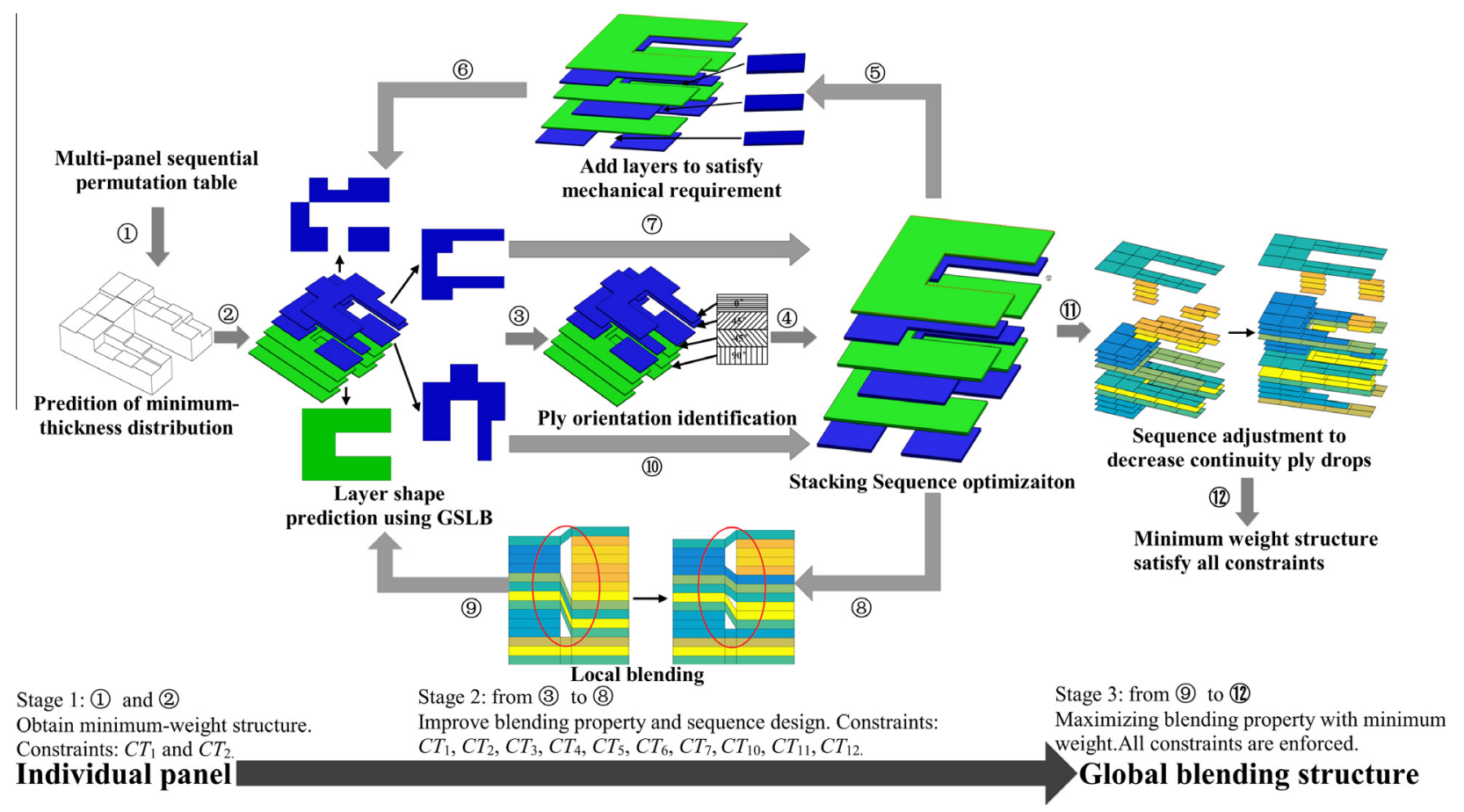

Fig. 4. Flowchart of the framework for design and optimization of tapered composite structures. 


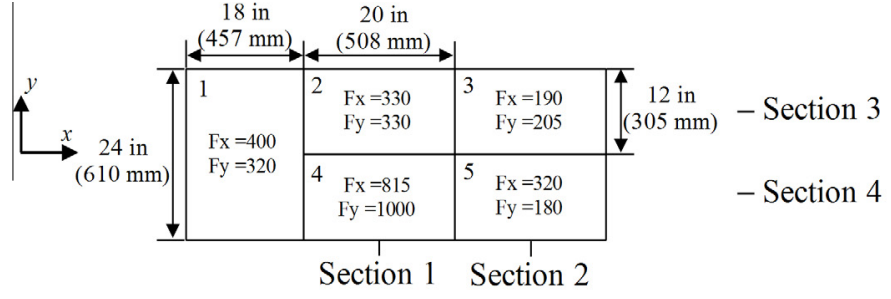

(a)

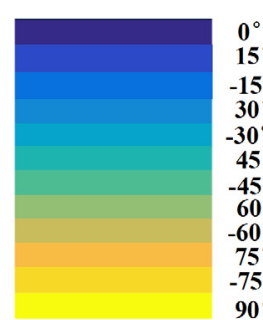

(b)

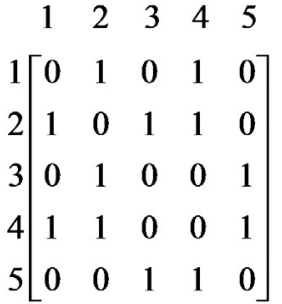

(c)

Fig. 5. 5-Panel example: (a) geometry, loads (all loads in lbf/in; 1lbf/in = 175.127 N/m) and definition of sections; (b) legend of ply orientations; (c) adjacent matrix.

\section{Detailed design techniques}

To illustrate the design techniques, a simple example with 5 panels is chosen for demonstration of the proposed framework, as shown in Fig. 5. Dimensions of panels and applied loads are shown in Fig. 5(a) with the legend for 12 candidate ply orientations $\left\{0^{\circ}, \pm 15^{\circ}, \pm 30^{\circ}, \pm 45^{\circ}, \pm 60^{\circ}, \pm 75^{\circ}, 90^{\circ}\right\}$ in Fig. 5(b). The adjacent matrix of 5 panels is given in Fig. 5(c). The material is graphite/ epoxy IM7/8552 with properties $E_{1}=141 \mathrm{GPa}$ (20.5 Msi), $E_{2}=9.03 \mathrm{GPa}(1.31 \mathrm{Msi}), G_{12}=4.27 \mathrm{GPa}(0.62 \mathrm{Msi})$ and $v_{12}=0.32$. Ply thickness is $t=0.191 \mathrm{~mm}$ (0.0075 in). In the following sections, the design process for the proposed framework is introduced.

\subsection{Multi-panel sequential permutation table}

The sequential permutation table (SPT) method was developed in [24]. It was observed that the $\lambda_{c b}$ can be expressed as a linear combination of the ply orientation based stacking sequences as shown in Eqs. (6) and (7); thus, superposition principle is suitable for evaluation of the $\lambda_{c b}$. Based on Eq. (4)-(7), the contribution of one lamina (symmetrical) to the $\lambda_{c b}$ can be evaluated without identifying a stacking sequence or a number of plies. Assume that there are $M$ candidate ply orientations $\left\{\theta_{1}, \theta_{2}, \ldots, \theta_{\mathrm{M}}\right\}$ and $P$ panels in total under compressive loads $\left(F_{x}, F_{y}\right)_{i}, i$ is the panel number, $1 \leqslant i \leqslant P$. Their buckling load factors are calculated, as shown in Table 1. Sort them from maximum to minimum, their corresponding ply orientations from the best to the worst are presented in the multi-panel sequential permutation table (MSPT), as shown in Table 2. The algorithm to obtain the MSPT is summarized in Algorithm 1.

These ply orientations satisfy: $\lambda_{c b}\left(\theta_{\max }^{i}\right)>\ldots>\lambda_{c b}\left(\theta_{j}^{i}\right)>\ldots>$ $\lambda_{c b}\left(\theta_{\min }^{i}\right), 1 \leqslant j \leqslant M$. $\lambda_{c b}(\theta)$ denotes the buckling load factor for one lamina $\theta$ (symmetrical). The MSPT provides a guidance in the stacking sequence design: the best ply orientation of each panel has a priority to be set in the laminate for the purpose of saving weight.

\section{Algorithm 1: MSPT}

(1) Initialize ply orientations $\left\{\theta_{1}, \theta_{2}, \ldots, \theta_{M}\right\}$;

(2) For $i=1: P$

For $j=1: M$

Calculate $\lambda_{c b}\left(\theta_{j}^{i}\right)$, and save it;

\section{End For}

\section{End For}

(3) Sort $\lambda_{c b}$ of each panel from the maximal to the minimal and re-write their corresponding ply orientations in the MSPT.

\subsection{Prediction of minimum-thickness distribution}

To obtain the minimum weight, the smaller the thickness of each panel the lighter the structure. Hence, it is preferable to use
Table 1

Buckling load factors for one lamina.

\begin{tabular}{lllll}
\hline One lamina & \multicolumn{4}{l}{ Panels under specific load cases $\left(F_{x}, F_{y}\right)_{i}$} \\
\cline { 2 - 5 } & 1 & 2 & $\ldots$ & $P$ \\
& $\left(F_{x}, F_{y}\right)_{1}$ & $\left(F_{x}, F_{y}\right)_{2}$ & $\ldots$ & $\left(F_{x}, F_{y}\right)_{P}$ \\
\hline$\left[\theta_{1}\right]_{s}$ & $\lambda_{c b}\left(\theta_{1}^{1}\right)$ & $\lambda_{c b}\left(\theta_{1}^{2}\right)$ & $\ldots$ & $\lambda_{c b}\left(\theta_{1}^{P}\right)$ \\
$\ldots$ & $\ldots$ & $\ldots$ & $\ldots$ & $\ldots$ \\
{$\left[\theta_{j}\right]_{s}$} & $\lambda_{c b}\left(\theta_{j}^{1}\right)$ & $\lambda_{c b}\left(\theta_{j}^{2}\right)$ & $\ldots$ & $\lambda_{c b}\left(\theta_{j}^{P}\right)$ \\
$\ldots$ & $\ldots$ & $\ldots$ & $\ldots$ & $\ldots$ \\
{$\left[\theta_{M}\right]_{s}$} & $\lambda_{c b}\left(\theta_{M}^{1}\right)$ & $\lambda_{c b}\left(\theta_{M}^{2}\right)$ & $\ldots$ & $\lambda_{c b}\left(\theta_{M}^{P}\right)$ \\
\hline $1 \leqslant j \leqslant M$ & & & &
\end{tabular}

Table 2

Multi-panel sequential permutation table (MSPT).

\begin{tabular}{lllll}
\hline Best ply orientation number & \multicolumn{4}{l}{ Panels under specific load cases $\left(F_{x}, F_{y}\right)_{i}$} \\
\cline { 2 - 5 } & 1 & 2 & $\ldots$ & $P$ \\
& $\left(F_{x}, F_{y}\right)_{1}$ & $\left(F_{x}, F_{y}\right)_{2}$ & $\ldots$ & $\left(F_{x}, F_{y}\right)_{P}$ \\
\hline 1 & $\theta_{\max }^{1}$ & $\theta_{\max }^{2}$ & $\ldots$ & $\theta_{\max }^{P}$ \\
$\ldots$ & $\ldots$ & $\ldots$ & $\ldots$ & $\ldots$ \\
$j$ & $\theta_{j}^{1}$ & $\theta_{j}^{2}$ & $\ldots$ & $\theta_{j}^{P}$ \\
$\ldots$ & $\ldots$ & $\ldots$ & $\ldots$ & $\ldots$ \\
$M$ & $\theta_{\min }^{1}$ & $\theta_{\min }^{2}$ & $\ldots$ & $\theta_{\min }^{P}$ \\
\hline
\end{tabular}

$1 \leqslant j \leqslant M$

the best ply orientation for each panel as presented in MSPT to obtain the minimum thickness without considering the blending requirement. However, the symmetry and balance constraints $C T 1$ and $C T 2$ are enforced. To satisfy the balance requirement $C T 2$, if the minimum ply number is an odd number, a better ply orientation of $0^{\circ}$ or $90^{\circ}$ (choose from MSPT) is used to replace the layer at the mid-plane of the laminate. The obtained minimumthickness distribution is shown in Table 3 with its figure shown in Fig. 6. The algorithm to predict the thickness is summarized in Algorithm 2, the variable 'thickness' is an array to record ply number of each panel.

Table 3

Minimum-thickness distribution.

\begin{tabular}{llll}
\hline $\begin{array}{l}\text { Panel } \\
\text { number }\end{array}$ & $\begin{array}{l}\text { Minimum thickness } \\
\text { stacking sequence }\end{array}$ & $\begin{array}{l}\text { Minimum } \\
\text { thickness }\end{array}$ & $\begin{array}{l}\text { Buckling load } \\
\text { factor }\end{array}$ \\
\hline 1 & {$\left[\left(\theta_{\max }^{1}\right)_{n 1}\right]_{s}$} & $n 1$ & $\lambda_{c b}\left(\left[\left(\theta_{\max }^{1}\right)_{n 1}\right]_{s}\right)$ \\
$\ldots$ & $\ldots$ & $\ldots$ & $\ldots$ \\
$i$ & {$\left[\left(\theta_{\max }^{i}\right)_{i} / 0\right]_{s}$} & $n i+1$ & $\lambda_{c b}\left(\left[\left(\theta_{\max }^{i}\right)_{n i} / 0\right]_{s}\right)$ \\
$\ldots$ & $\ldots$ & $\ldots$ & $\ldots$ \\
$P$ & {$\left[\left(\theta_{\max }^{P}\right)_{n P}\right]_{s}$} & $n P$ & $\lambda_{c b}\left(\left[\left(\theta_{\max }^{P}\right)_{n P}\right]_{s}\right)$ \\
\hline
\end{tabular}

ni or $n i+1(1 \leqslant i \leqslant P)$ are one half the number of total plies of panel $i$. 

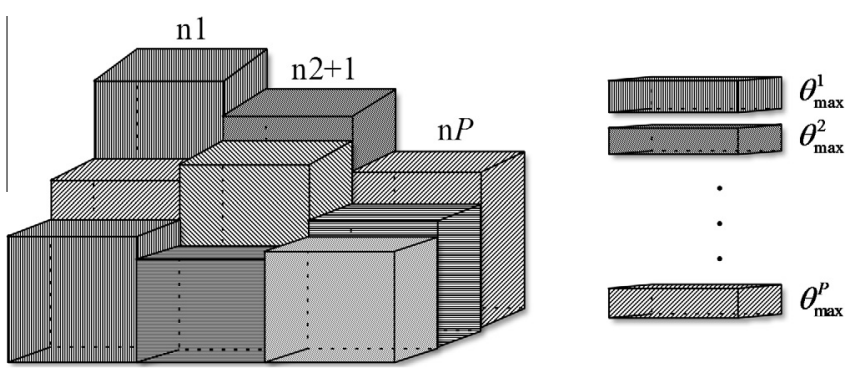

Fig. 6. Demonstration of minimum-thickness distribution.

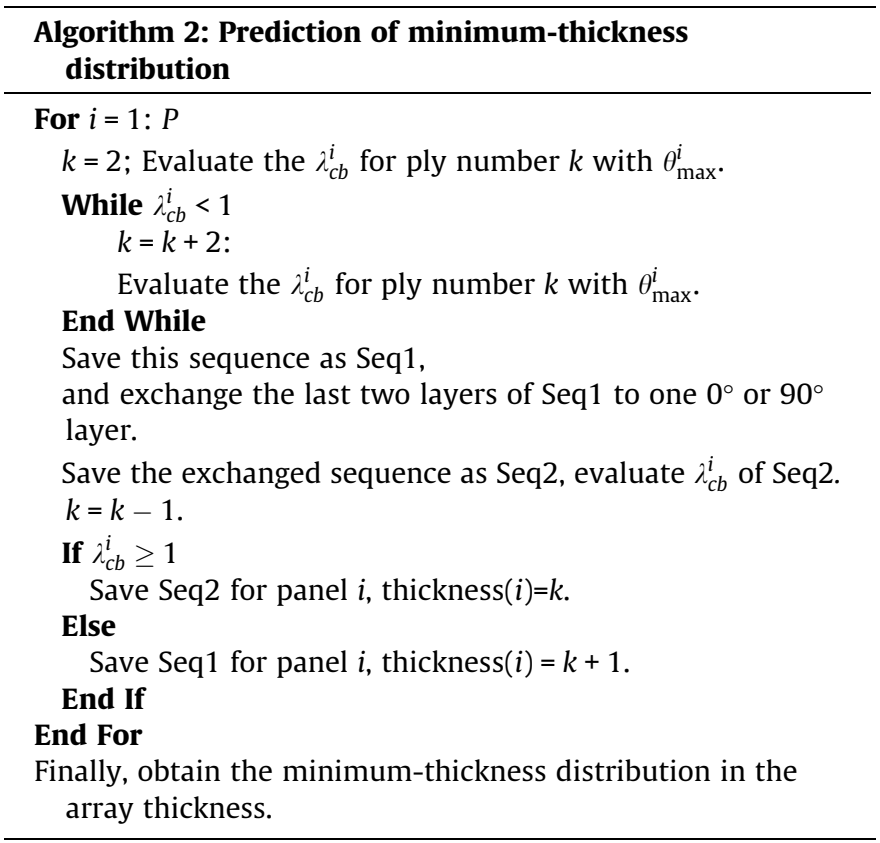

\subsection{Global shared-layer blending method}

The global shared-layer blending (GSLB) method was first introduced in [24]; it was developed to predict the layer shape of the tapered composite structure according to its thickness distribution. However, the GSLB method can only be applied to the same ply orientation. Thus, assume that all layers have the same ply orientation in minimum-thickness distribution (Table 3), the GSLB is applied to obtain the shape of the shared layers, in which the constraints CT1 and CT12 are enforced. The detailed description of GSLB is provided in [24], here, the summarised algorithm is given in Algorithm 3. The shared layer covers all panels is defined as the global shared layer; on the contrary, the layer not covers all panels is defined as the subregion shared layer [24].

\section{Algorithm 3: GSLB}

(1) Input the thickness distribution of multiple ply orientations.

(2) For a thickness distribution of one ply orientation, obtain the minimum number of all panels. All panels that contain the ply orientation minus the minimum number, the set of panels contain the minimum number of ply orientation assemble the shared layer.
(3) Assess the distribution of the shared layer (the panels contain minimum number), and get different layer shape of the shared layers. Save the shared layers.

(4) Delete the minimum number of plies from the panels of the thickness distribution and save the rest of thickness distribution of the ply orientation.

(5) Repeat (2) to (4) until all plies are deleted from the thickness distribution.

(6) Apply (2) to (5) to various ply orientations, obtain the shape of shared layers with different ply orientations.

\subsection{Ply-orientation identification}

Ply orientations should be identified based on the shape of shared layer, stacking position and the buckling load factor of the shared layer. In this section, a new parameter is introduced: $C_{f}-$ the contribution factor is defined to characterize the contribution of a shared layer to the overall structure:

$$
\begin{aligned}
& C_{f}=\frac{1}{R} \sum_{i=1}^{R} \frac{S_{i} \lambda_{c b}^{i}}{S} ; \\
& S=\sum_{i=1}^{R} S_{i}
\end{aligned}
$$

where, $C_{f}$ is the contribution factor of the shared layer, $R$ is the total number of panels covered by the shared layer, $S_{i}$ is the area of panel $i$ covered by the shared layer, $S$ is the total area of the shared layer, $\lambda_{c b}^{i}$ is the buckling load factor of panel $i$. The angle maximizes $C_{f}$ should be set as the ply orientation of the shared layer. However, the stacking position of the shared layer should be identified for evaluation of $\lambda_{c b}^{i}$. An explanation of the definition of the contribution factor is introduced as follows, the identification process of the ply orientation is given later.

Assume that there are two panels $i$ and $j$ with areas $S_{i}$ and $S_{j}$, and $S_{i}>S_{j}, S=S_{i}+S_{j}$, for the two candidate ply orientations $\theta_{1}$ and $\theta_{2}$, the contribution factors $C_{f}$ are

$$
\begin{aligned}
& C_{f}\left(\theta_{1}\right)=\frac{S_{i}}{2 S} \lambda_{c b}^{i}\left(\theta_{1}\right)+\frac{S_{j}}{2 S} \lambda_{c b}^{j}\left(\theta_{1}\right) ; \\
& C_{f}\left(\theta_{2}\right)=\frac{S_{i}}{2 S} \lambda_{c b}^{i}\left(\theta_{2}\right)+\frac{S_{j}}{2 S} \lambda_{c b}^{j}\left(\theta_{2}\right) ; \text { thus, } \\
& C_{f}\left(\theta_{1}\right)-C_{f}\left(\theta_{2}\right)=\frac{S_{i}}{2 S}\left(\lambda_{c b}^{i}\left(\theta_{1}\right)-\lambda_{c b}^{i}\left(\theta_{2}\right)\right)+\frac{S_{j}}{2 S}\left(\lambda_{c b}^{j}\left(\theta_{1}\right)-\lambda_{c b}^{j}\left(\theta_{2}\right)\right)
\end{aligned}
$$

To select a suitable ply orientation for the shared layer, the relationships for $\lambda_{c b}^{i}$ should be analyzed:
(1) $\underline{\lambda_{c b}^{i}\left(\theta_{1}\right)>\lambda_{c b}^{i}\left(\theta_{2}\right)}, \lambda_{c b}^{j}\left(\theta_{1}\right)>\lambda_{c b}^{j}\left(\theta_{2}\right)$;
(2) $\underline{\lambda_{c b}^{i}\left(\theta_{1}\right)<\lambda_{c b}^{i}\left(\theta_{2}\right)}, \lambda_{c b}^{j}\left(\theta_{1}\right)<\lambda_{c b}^{j}\left(\theta_{2}\right)$;
(3) $\lambda_{c b}^{i}\left(\theta_{1}\right)>\lambda_{c b}^{i}\left(\theta_{2}\right), \lambda_{c b}^{j}\left(\theta_{1}\right)<\lambda_{c b}^{j}\left(\theta_{2}\right)$;

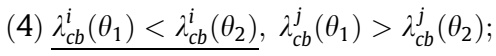

According to Eqs. (10) and (11): the ply orientation of the shared layer is depending on the value of $C_{f}\left(\theta_{1}\right)-C_{f}\left(\theta_{2}\right)$ :

In case ( 1$), \theta_{1}$ should be set as the shared layer's ply orientation. In case (2), $\theta_{2}$ should be set as the shared layer's ply orientation.

In case (3), since $S_{i} / S>S_{j} / S$, the positive value $\lambda_{c b}^{i}\left(\theta_{1}\right)-\lambda_{c b}^{i}\left(\theta_{2}\right)$ is amplified and the negative value $\lambda_{c b}^{j}\left(\theta_{1}\right)-\lambda_{c b}^{j}\left(\theta_{2}\right)$ is diminished. In most cases, $\lambda_{c b}^{i}\left(\theta_{1}\right)$ is close to $\lambda_{c b}^{i}\left(\theta_{2}\right)$ and $\lambda_{c b}^{j}\left(\theta_{1}\right)$ is close to $\lambda_{c b}^{j}\left(\theta_{2}\right)$, thus $C_{f}\left(\theta_{1}\right)>C_{f}\left(\theta_{2}\right)$ is a common situation. For the shared layer it is preferable to choose $\theta_{1}$ as its ply orientation. 
In case (4), since $S_{i} / S>S_{j} / S$, the negative value $\lambda_{c b}^{i}\left(\theta_{1}\right)-\lambda_{c b}^{i}\left(\theta_{2}\right)$ is amplified and the positive value $\lambda_{c b}^{j}\left(\theta_{1}\right)-\lambda_{c b}^{j}\left(\theta_{2}\right)$ is diminished. In most cases, $\lambda_{c b}^{i}\left(\theta_{1}\right)$ is close to $\lambda_{c b}^{i}\left(\theta_{2}\right)$ and $\lambda_{c b}^{j}\left(\theta_{1}\right)$ is close to $\lambda_{c b}^{j}\left(\theta_{2}\right)$, thus $C_{f}\left(\theta_{1}\right)<C_{f}\left(\theta_{2}\right)$ is a common situation. For the shared layer it is preferable to choose $\theta_{2}$ as its ply orientation.

From the analysis above, it can be observed from the parts underlined in Eq. (11) that it is always preferable for the shared layer to choose the ply orientation with a larger $\lambda_{c b}$ belonging to the panel with a larger area. Moreover, for the subsequent design process, panels with smaller areas tend to have smaller $\lambda_{c b}$ (even the sequence change). When layers should be added to panels to increase $\lambda_{c b}$, the panel with a smaller area has priority for adding layers. Hence, ply orientations of the shared layers have more contributions to the panels with large areas, and the definition of Eq. (9) tends to choose the suitable ply orientation of a shared layer for saving weight.

In this section, the constraints $C T 1, C T 2$ and $C T 12$ are enforced, the disorientation requirement CT4 is considered for global shared layers. The selection of ply orientation for shared layers is introduced as follows:

- Step 1: According to the minimum-thickness distribution, in each panel, set the best ply orientation at all stacking positions. Sort the shared layers from maximum to minimum (according to their areas).

- Step 2: Use the biggest shared layer to replace the best ply orientation of each panel from the outermost position to the midplane (Fig. 7). After each replacement, evaluate $C_{f}$ for all candidate ply orientations from $\theta_{1}$ to $\theta_{\mathrm{M}}$. Save the ply orientation $\theta$ with the maximum $C_{f}$ for the shared layer. If the shared layer is a subregion shared layer, stacking position $k=k-1$ for each panel; otherwise, for a global shared layer, set another global shared layer with $-\theta$ at the next stacking position $k=k-1$, then stacking position $k=k-2$ for each panel. Reset ply orientation as the best ply orientation of each panel. Then use the next shared layer for ply-orientation identification. Repeat step 2 until all ply orientations of shared layers are identified.

- Step 3: Satisfy the disorientation requirement for the global shared layers. For the global shared layers with ply orientation $\pm \theta(|\theta| \geqslant 30), 0^{\circ}$ or $90^{\circ}$ layer should be inserted between $+\theta$ and $-\theta$. Thus, replace the global shared layer with $0^{\circ}$ or $90^{\circ}$ layers (according to $C_{f}$ ). If the number of the global shared layer is odd, only one global shared layer should be replaced; otherwise, two adjacent layers should be replaced.

- Step 4: Satisfy the balance requirement for the subregion shared layers, the ply number of minimum-thickness distribution minus the number of global shared layers in each panel.
For a specific panel, if the rest of the ply number is even, set the best ply orientations $+\theta_{\max }$ and $-\theta_{\max }$ each half in this panel; Otherwise, set one $0^{\circ}$ or $90^{\circ}$ ply first (better one), and the rest of plies with the best ply orientations $+\theta_{\max }$ and $-\theta_{\max }$ each half in the panel.

- Step 5: Input the adjusted number of ply orientations of all panels into the GSLB algorithm to get the shared layers.

The detailed algorithms are demonstrated in Algorithm 4 and Algorithm 5.

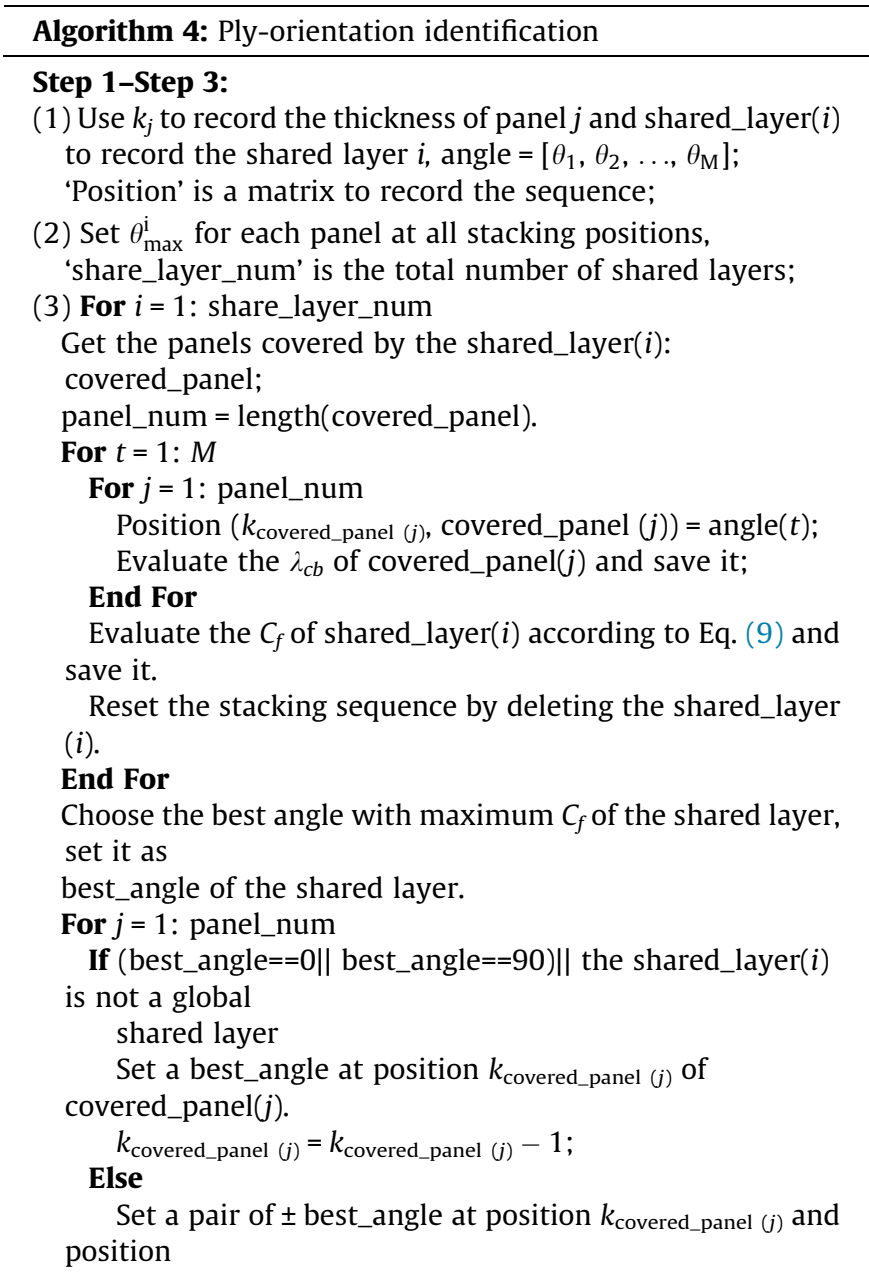

(continued on next page)

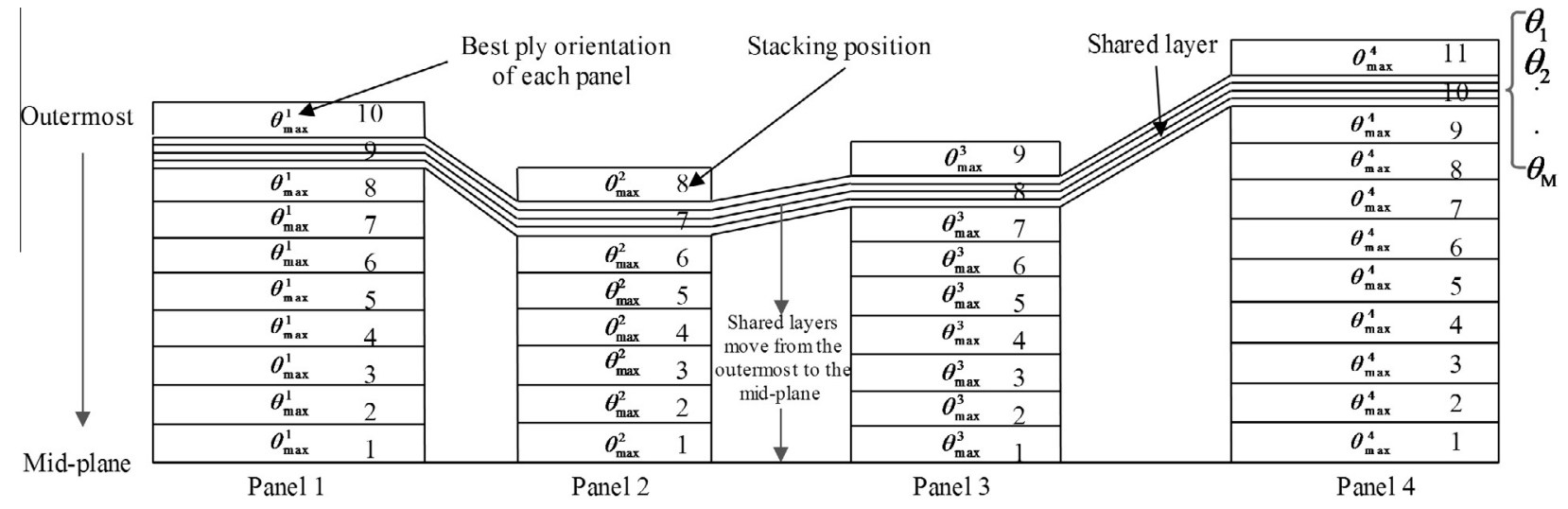

Fig. 7. Identification for ply orientation of shared layer. 


\author{
$k_{\text {covered_panel }(j)}-1$ of covered_panel $(j)$ to satisfy the \\ balance \\ requirement. \\ $k_{\text {covered_panel }(j)}=k_{\text {covered_panel }(j)}-2$; \\ End If \\ End For \\ End For
}

(4) Save the numbers of ply orientations for each panel in a matrix: region_content.

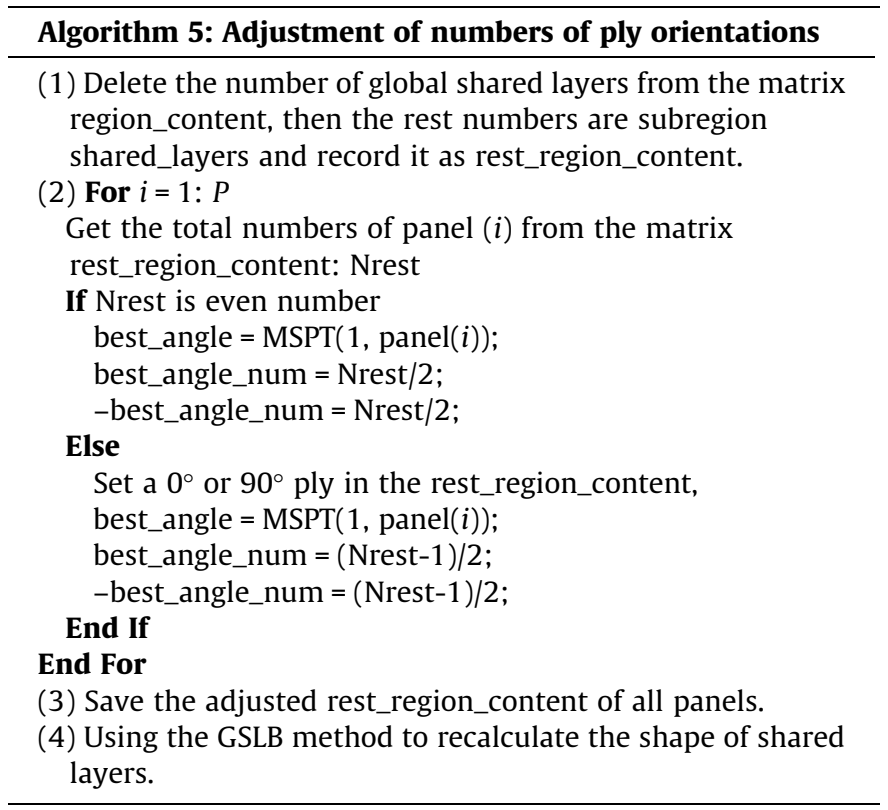

\subsection{Sequence optimization}

The ply orientation of each shared layer is identified in Section 3.4; sequences of these shared layers should be optimized. In this section, constraints $C T 1, C T 2, C T 3, C T 4, C T 5, C T 6, C T 7, C T 10$, CT11 and CT12 are imposed. First of all, the sequences of global shared layers are evaluated by full permutation; then, those sequences that satisfy the contiguity and disorientation requirements are selected as initial frame sequences for detailed sequence design. Then, the subregion shared layers are inserted from the maximal to the minimal (by area) with stacking positions from the outermost position to the mid-plane (see Fig. 8). The main concern is the influence of sequence on the $\lambda_{c b}$ of the shared layer; hence, the average buckling load factor is used to evaluate the best stacking position of the insert shared layer:

$C_{\text {ave }}=\frac{1}{R} \sum_{i=1}^{R}\left(\lambda_{c b}^{i}\right)$

where $R$ is the total number of panels covered by the shared layer, $\lambda_{c b}^{i}$ is the buckling load factor of panel $i$. $C_{a v e}$ is the average buckling load factor of the shared layer. The detailed steps of the sequence optimization strategy is introduced as follows:

Step 1: A full permutation of global shared layers, select the sequences satisfy the contiguity and disorientation requirements as initial frame sequences. Sort the subregion shared layers from the maximal to the minimal according to their areas.
Step 2: Use the first initial frame sequence for insert operation. Insert the maximal subregion shared layer from the outermost position to the mid-plane: for every insert position, if any panel of the shared layer violates individual panel's constraints, jump to the next insert position. After each insert operation, evaluate the $C_{a v e}$ of the subregion shared layer and save it, then delete the insert subregion shared-layer.

Step 3: For each subregion shared layer, choose the insert stacking position with the maximal $C_{a v e}$. Save the sequence for the insert operation of the next subregion shared layer. Repeat step 2 to step 3 until all subregion shared layers are inserted. Use the next initial frame sequence for insert operation, turn to step 2 . Step 4: Repeat step 2 to step 3 until all candidate initial frame sequences of global shared layers are used for sequence optimization. Choose the solution with the minimum number of panels violating buckling constraint for further design.

The detailed algorithm of the sequence optimization strategy is described in Algorithm 6.

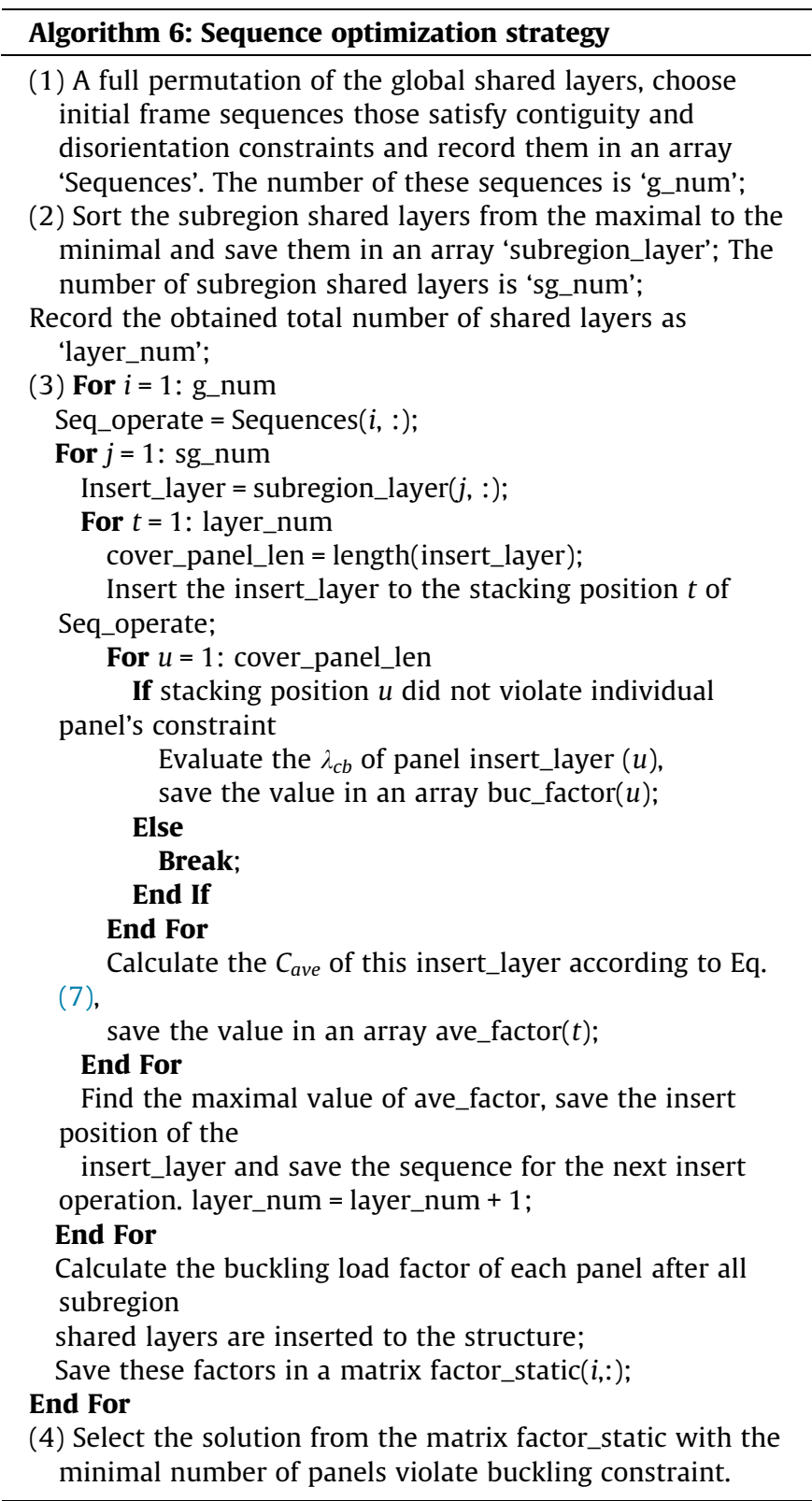




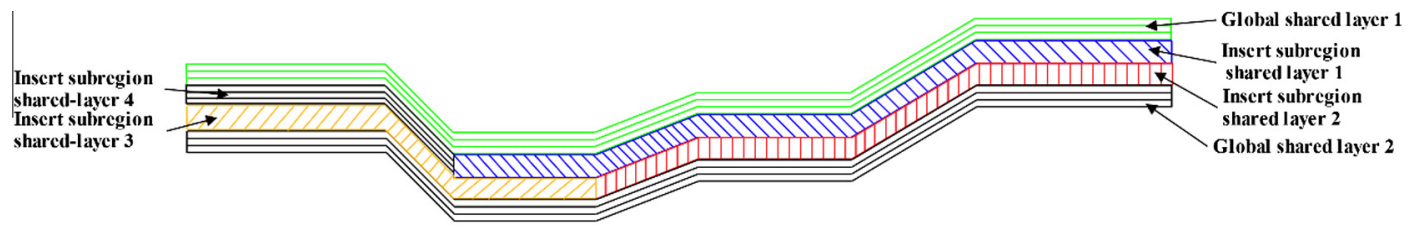

Fig. 8. Insert subregion shared layers to the global shared layers.

\subsection{Add layer}

The objective is to find a solution with the fewest layers to add for saving weight. An add layer strategy is developed, with the blending and lightweight requirements considered in design. In previous design, best ply orientations are set in individual panels except for the global shared layers. Even the $\lambda_{c b}$ is smaller than 1, it is very close to 1 . Thus, adding one layer is enough to satisfy buckling constraint in most panels. When the thickness and sequence are identified in a panel, the ply orientation of the added layer has a minor influence on $\lambda_{c b}$. As a result, when layers are added to the panel, the blending property should be satisfied in advance, followed by the individual panel's constraints. Detailed steps of the add-layer strategy is introduced as follows:

Step 1: The obtained sequence has the minimal number of panels violate the buckling constraint. Record the number of these panels in an array 'vio_panels'.

Step 2: In the array 'vio_panels', add one layer to each panel from the first to the last. The panel that selected for the addlayer operation is set as current panel. For the current panel: first choose a layer $0^{\circ}$ or $90^{\circ}$ (better one according to the MSPT) for the add-layer operation. Record the ply orientation of the added layer as add_angle.

Step 3: Detect the layers of current panel and its adjacent panels from the outermost position to the mid-plane.

Assessment 1: Do these panels have the same ply orientation add_angle? If yes, go to Assessment 2. If not, the add_angle layer will be added to the current panel as a single layer at the outermost position to maximize $\lambda_{c b}$, turn to Step 5 .

Assessment 2: Does the current panel have this layer at the same position? If not, add the layer add_angle at this position, turn to Step 5. If yes, turn to Step 4 .

Step 4: Delete the layer at this position. Choose the best $\pm \theta$ from the MSPT. Set add_angle $1=+\theta$ and add_angle $2=-\theta$. Repeat step 3 two times for these two angles. Try to delete a $0^{\circ}$ or $90^{\circ}$ layer from the current panel without violation of constraints.

Step 5: Evaluate the $\lambda_{c b}$ of the current panel to confirm that the add-layer operation is successful. Update the numbers of ply orientations in the current panel. Move to the next panel for add-layer operation, repeat step 2 to step 5 until all panels in the array vio_panels satisfy the buckling constraint.

Step 6: Update the numbers of ply orientations. Perform the GSLB method and sequence optimization strategy to optimize the structure.

The reason that the GSLB method is used again to evaluate the layer shape is that when one layer is added, it may connect to one shared layer or two separated shared layers, as shown in Fig. 9. Since the layer shape is changed, the sequence should be rearranged to ensure the satisfaction of constraints. Eventually, an optimal sequence with minimum weight, which satisfies all constraints is obtained. The algorithm of the add layer strategy is summarized in Algorithm 7.

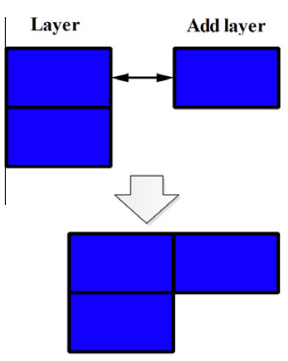

(a)

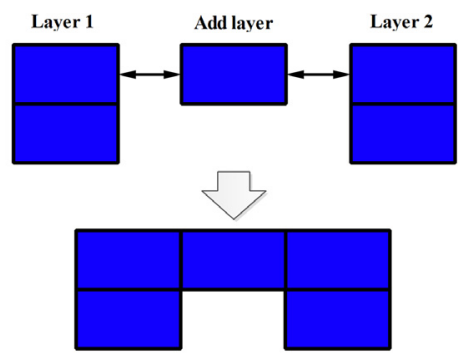

(b)
Fig. 9. Add layer and blend to shared layers: (a) blending with one shared layer (b) blending with two separated shared layers.

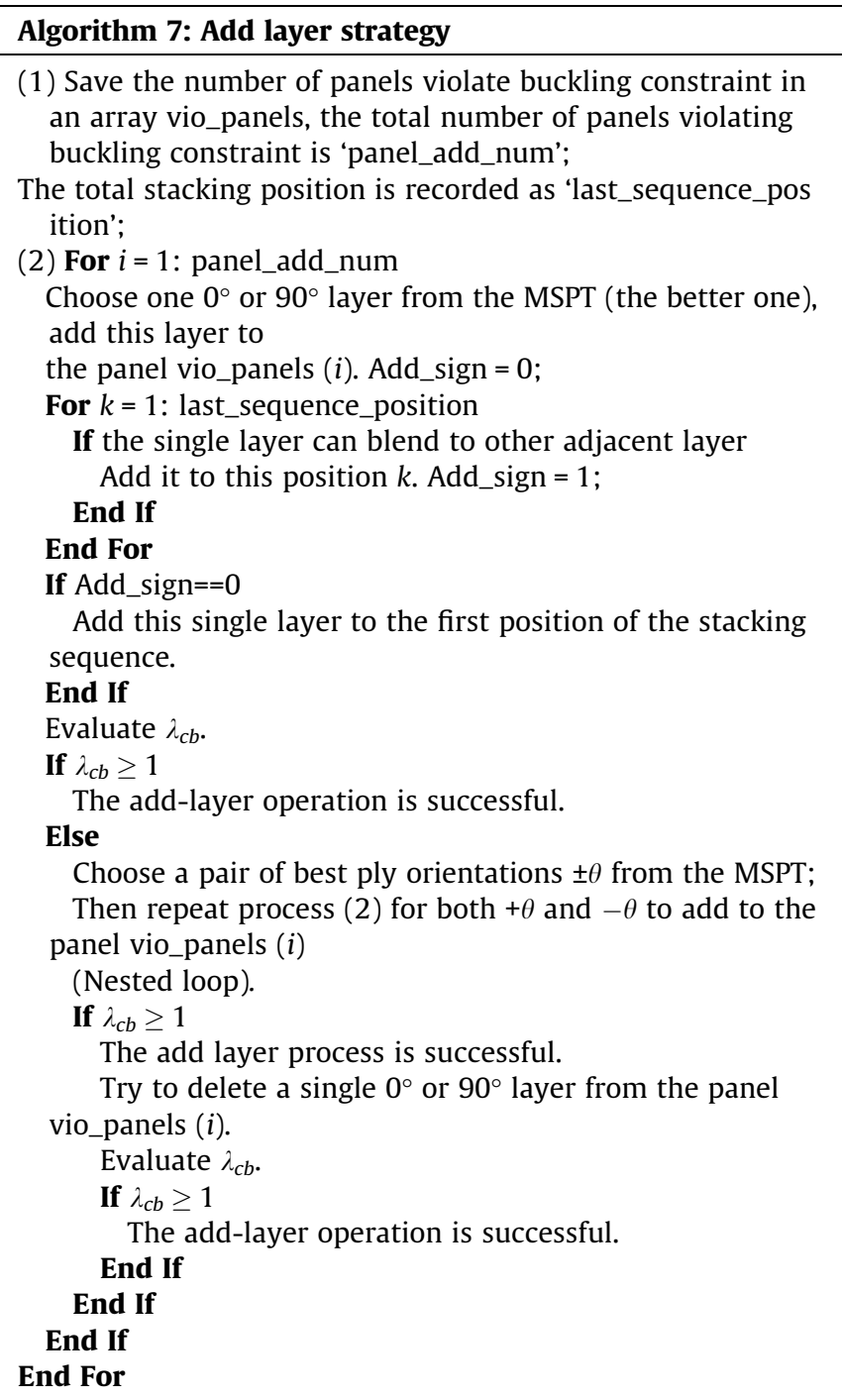




\subsection{Local blending}

In this section, the ply orientations of each panel will be adjusted to improve the blending property; the rest of constraints in Eq. (3) CT8, CT9 and CT13 are enforced in this step. In Section 3.4, the best ply orientations are chosen from the MSPT except for the global shared layers. From the previous analysis, the way to choose the ply orientation may lead to big margins for the $\lambda_{c b}$ with a bad blending property (ply drops between adjacent panels due to a mismatch of best ply orientations, see Fig. 10). To improve the blending property, ply orientations should be adjusted. The detailed algorithm for ply orientations adjustment is summarized in Algorithm 8.

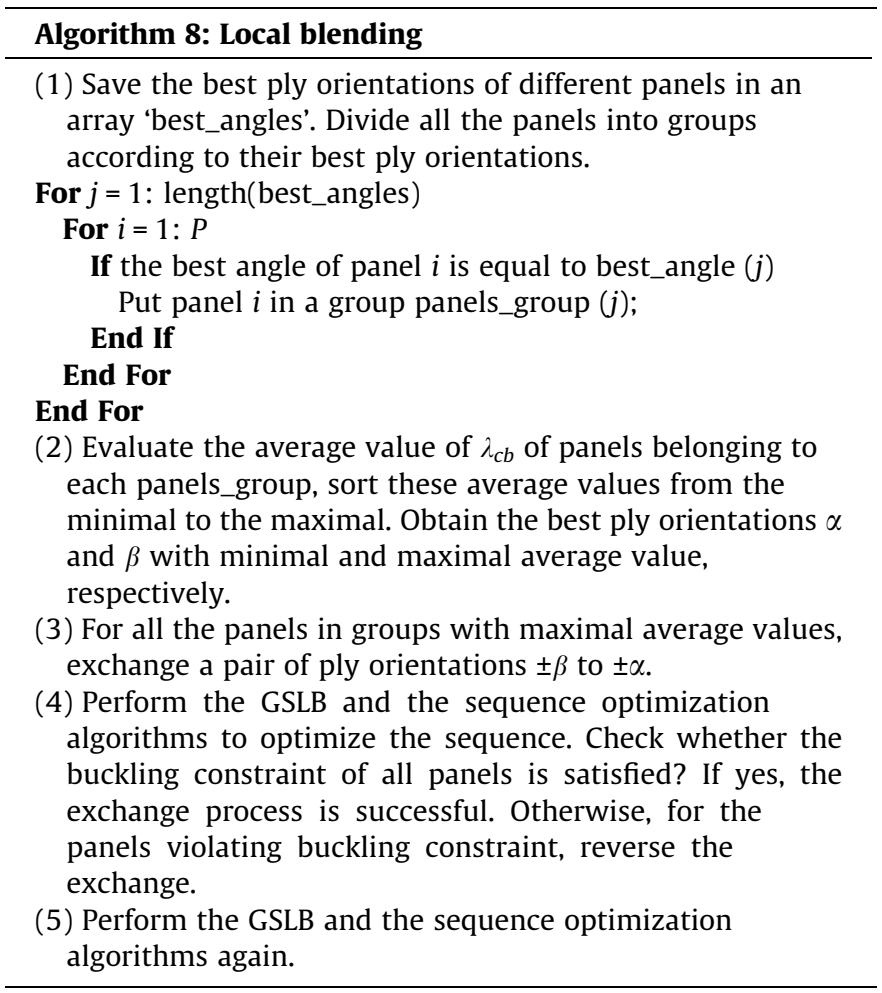

(a)

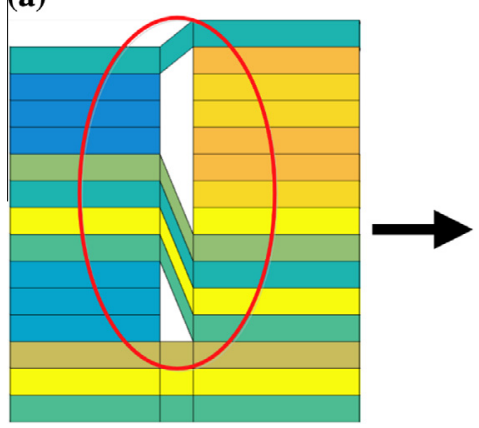

(b)

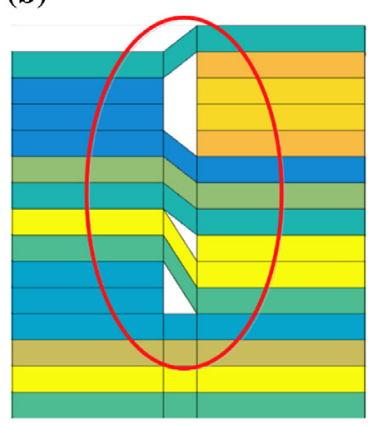

Fig. 10. Local blending by exchanging ply orientations.

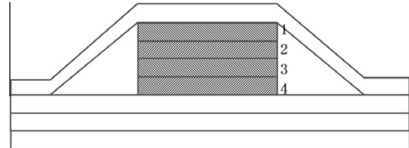

(a)

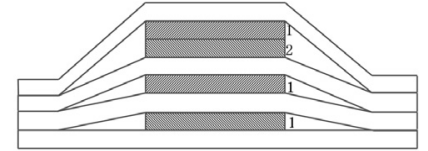

(b)
Fig. 11. Definition of continuity ply drops: (a) continuity ply drop number; (b) decrease the continuity ply drop numbers.

\subsection{Sequence adjustment}

In previous design, the subregion shared layers with best ply orientations are often stacked at the outermost positions to maintain the buckling load factors. However, this arrangement leads to several subregion shared layers or single layers stacked together and causes continuity ply drops in the structure. The definition of continuity ply drops is shown in Fig. 11. To decrease the continuity ply drops, the subregion shared layers should be arranged between two large shared layers to smooth the load path, such as sandwich structures (Fig. 11(b)). At the same time, constraints should not be violated when adjusting the sequence of subregion shared layers. The detailed sequence adjustment process is introduced as follows, Algorithm 9 gives the detailed execution process.

Step 1: Assume that the number of shared layers is share num. From the outermost position to the mid-plane, choose the first subregion shared layer as the current shared layer. Delete this shared layer from its original stacking position. After deletion, ensure that the sequence does not violate any constraints, save the sequence as Origional_ sequence.

Step 2: Insert this current shared layer from the outermost to the mid-plane (except for the original position), assume that the insert position is $k$.

Step 3: Check the sequence of each panel covered by the current shared layer,

Assessment 1: Does the sequence violate the contiguity or disorientation constraints? If yes, jump to the next insert position; Otherwise, evaluate the $\lambda_{c b}$ for each panel covered by the current shared layer.

Assessment 2: Are the buckling constraint of panels covered by the current shared layer satisfied? If yes, save this sequence and get the ply drop numbers of the layers up and down of this shared layer, turn to Step 4; Otherwise, jump to the next insert position, turn to Step 3.

Step 4: Save the $\lambda_{c b}$ and continuity ply-drop numbers (the continuity ply-drop number is related to up and down shared layers of the current shared layer) of the shared layer, save the sequence. Reset the sequence as Origional_sequence, $k=k+1$; repeat Step 2 to Step 4 utill $k=$ share_num.

Step 5: Choose the insert position with minimal continuity ply-drop numbers, save the sequence as Optimal_sequence for next shared layer adjustment; this shared layer will not be rearranged in further adjustments. Then move to the next subregion shared layer for sequence adjustment operation. Repeat Step 1 to Step 5 until all subregion shared layers are adjusted. 
(a) Buckling load factors $\left(1 \times 10^{-4}\right)$ for one lamina in the 5-panel problem

\begin{tabular}{llllll}
\hline \multirow{2}{*}{ One lamina } & \multicolumn{6}{c}{ Buckling load factors $\left(1 \times 10^{-4}\right)$} \\
\cline { 2 - 6 } & $\mathbf{1}$ & $\mathbf{2}$ & 3 & 4 & $\mathbf{5}$ \\
\hline$[0] \mathrm{s}$ & $\mathbf{3 . 3 9}$ & $\mathbf{2 . 2 3}$ & 3.66 & $\mathbf{0 . 7 7}$ & $\mathbf{3 . 3 9}$ \\
{$[15] \mathrm{s}$} & $\mathbf{3 . 6 1}$ & 3.23 & $\mathbf{5 . 3 0}$ & $\mathbf{1 . 1 2}$ & $\mathbf{4 . 9 1}$ \\
{$[-15] \mathrm{s}$} & $\mathbf{3 . 6 1}$ & $\mathbf{3 . 2 3}$ & $\mathbf{5 . 3 0}$ & $\mathbf{1 . 1 2}$ & $\mathbf{4 . 9 1}$ \\
{$[30] \mathrm{s}$} & $\mathbf{3 . 9 6}$ & $\mathbf{5 . 5 8}$ & $\mathbf{9 . 1 6}$ & $\mathbf{1 . 9 4}$ & $\mathbf{8 . 4 8}$ \\
{$[-30] \mathrm{s}$} & $\mathbf{3 . 9 6}$ & $\mathbf{5 . 5 8}$ & $\mathbf{9 . 1 6}$ & $\mathbf{1 . 9 4}$ & $\mathbf{8 . 4 8}$ \\
{$[45] \mathrm{s}$} & $\mathbf{3 . 8 3}$ & $\mathbf{7 . 9 0}$ & $\mathbf{1 2 . 9 7}$ & $\mathbf{2 . 7 4}$ & $\mathbf{1 2 . 0 1}$ \\
{$[-45] \mathrm{s}$} & $\mathbf{3 . 8 3}$ & $\mathbf{7 . 9 0}$ & $\mathbf{1 2 . 9 7}$ & $\mathbf{2 . 7 4}$ & $\mathbf{1 2 . 0 1}$ \\
{$[60]$ s } & $\mathbf{2 . 9 9}$ & $\mathbf{9 . 1 9}$ & $\mathbf{1 5 . 0 9}$ & $\mathbf{3 . 1 9}$ & $\mathbf{1 3 . 9 7}$ \\
{$[-60] \mathrm{s}$} & $\mathbf{2 . 9 9}$ & $\mathbf{9 . 1 9}$ & $\mathbf{1 5 . 0 9}$ & $\mathbf{3 . 1 9}$ & $\mathbf{1 3 . 9 7}$ \\
{$[75] \mathrm{s}$} & $\mathbf{1 . 9 2}$ & $\mathbf{9 . 4 8}$ & $\mathbf{1 5 . 5 7}$ & 3.29 & $\mathbf{1 4 . 4 2}$ \\
{$[-75]$ s } & $\mathbf{1 . 9 2}$ & $\mathbf{9 . 4 8}$ & $\mathbf{1 5 . 5 7}$ & $\mathbf{3 . 2 9}$ & $\mathbf{1 4 . 4 2}$ \\
{$[90] \mathrm{s}$} & $\mathbf{1 . 4 4}$ & $\mathbf{9 . 4 5}$ & $\mathbf{1 5 . 5 1}$ & 3.28 & $\mathbf{1 4 . 3 7}$ \\
\hline
\end{tabular}

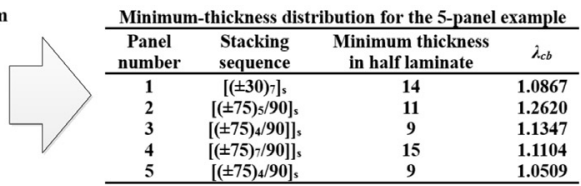

Multi-panel sequential permutation table for the 5-panel problem Optimal number Ply orientations: from best to worst

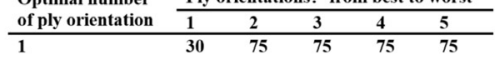

\begin{tabular}{llllll}
\hline 1 & 30 & 75 & 75 & 75 & 75 \\
2 & -30 & -75 & -75 & -75 & -75 \\
3 & 45 & 90 & 90 & 90 & 90 \\
4 & -45 & 60 & 60 & 60 & 60 \\
5 & 15 & -60 & -60 & -60 & -60 \\
6 & -15 & 45 & 45 & 45 & 45 \\
7 & 0 & -45 & -45 & -45 & -45 \\
8 & 60 & 30 & 30 & 30 & 30 \\
9 & -60 & -30 & -30 & -30 & -30 \\
10 & 75 & 15 & 15 & 15 & 15 \\
11 & -75 & -15 & -15 & -15 & -15 \\
12 & 90 & 0 & 0 & 0 & 0 \\
\hline
\end{tabular}

The best ply orientation is the first row with number 1 ,

(2) Prediction of minimum-thickness distribution

(1) Multi-panel sequential permutation table

\begin{tabular}{|c|c|c|c|c|c|c|}
\hline \multirow{2}{*}{$\begin{array}{l}\text { Shared layer } \\
\text { type }\end{array}$} & \multirow{2}{*}{$\begin{array}{l}\text { Shared layer } \\
\text { number }\end{array}$} & \multicolumn{5}{|c|}{ Panels covered by shared layers } \\
\hline & & 1 & 2 & 3 & 4 & 5 \\
\hline Global & 1 & 1 & 2 & 3 & 4 & 5 \\
\hline \multirow[t]{8}{*}{ shared layers } & 2 & 1 & 2 & 3 & 4 & 5 \\
\hline & 3 & 1 & 2 & 3 & 4 & 5 \\
\hline & 4 & 1 & 2 & 3 & 4 & 5 \\
\hline & 5 & 1 & 2 & 3 & 4 & 5 \\
\hline & 6 & 1 & 2 & 3 & 4 & 5 \\
\hline & 7 & 1 & 2 & 3 & 4 & 5 \\
\hline & 8 & 1 & 2 & 3 & 4 & 5 \\
\hline & 9 & 1 & 2 & 3 & 4 & 5 \\
\hline & 10 & 1 & 2 & & 4 & \\
\hline \multirow{5}{*}{ shared layers } & 11 & 1 & 2 & & 4 & \\
\hline & 12 & 1 & & & 4 & \\
\hline & 13 & 1 & & & 4 & \\
\hline & 14 & 1 & & & 4 & \\
\hline & 15 & & & & 4 & \\
\hline Minimum thic & ss of panels & 14 & 11 & 9 & 15 & 9 \\
\hline
\end{tabular}

(3) Global shared-layer blending method

\begin{tabular}{|c|c|c|c|c|c|c|c|c|c|c|c|c|c|}
\hline \multirow{3}{*}{$\begin{array}{c}\begin{array}{c}\text { Shared } \\
\text { layer } \\
\text { number }\end{array} \\
\end{array}$} & \multicolumn{12}{|c|}{ Selection of ply orientations for the 5-panel problem } & \multirow{3}{*}{$-\underset{\text { Prientation }}{\text { Ply }}$} \\
\hline & 1 & 2 & 3 & 4 & 5 & 6 & 7 & 8 & 9 & 10 & 11 & 12 & \\
\hline & $0^{\circ}$ & $15^{\circ}$ & $-15^{\circ}$ & $30^{\circ}$ & $-30^{\circ}$ & $45^{\circ}$ & $-45^{\circ}$ & $60^{\circ}$ & $-60^{\circ}$ & $75^{\circ}$ & $-75^{\circ}$ & $90^{\circ}$ & \\
\hline 1,2 & 0.9588 & 0.9839 & 0.9839 & 1.0400 & 1.0400 & 1.0872 & 1.0872 & 1.1004 & 1.1004 & 1.0885 & 1.0885 & 1.0796 & $60,-60$ \\
\hline 3,4 & 1.0184 & 1.0346 & 1.0346 & 1.0706 & 1.0706 & 1.1002 & 1.1002 & 1.1072 & 1.1072 & 1.0980 & 1.0980 & 1.0916 & $60,-60$ \\
\hline 5 & 1.0639 & 1.0733 & 1.0733 & 1.0939 & 1.0939 & 1.1102 & 1.1102 & 1.1128 & 1.1128 & 1.1060 & 1.1060 & 1.1017 & 90 \\
\hline 6,7 & 1.0815 & 1.0882 & 1.0882 & 1.1028 & 1.1028 & 1.1140 & 1.1140 & 1.1151 & 1.1151 & 1.1094 & 1.1094 & 1.1060 & $60,-60$ \\
\hline 8,9 & 1.1061 & 1.1091 & 1.1091 & 1.1152 & 1.1152 & 1.1194 & 1.1194 & 1.1187 & 1.1187 & 1.1151 & 1.1151 & 1.1131 & $45,-45$ \\
\hline 10 & 1.1301 & 1.1317 & 1.1317 & 1.1351 & 1.1351 & 1.1371 & 1.1371 & 1.1360 & 1.1360 & 1.1332 & 1.1332 & 1.1318 & 45 \\
\hline 11 & 1.1339 & 1.1348 & 1.1348 & 1.1368 & 1.1368 & 1.1380 & 1.1380 & 1.1372 & 1.1372 & 1.1355 & 1.1355 & 1.1347 & 45 \\
\hline 12 & 1.0912 & 1.0919 & 1.0919 & 1.0934 & 1.0934 & 1.0943 & 1.0943 & 1.0939 & 1.0939 & 1.0927 & 1.0927 & 1.0921 & 45 \\
\hline 13 & 1.0932 & 1.0936 & 1.0936 & 1.0943 & 1.0943 & 1.0948 & 1.0948 & 1.0947 & 1.0947 & 1.0943 & 1.0943 & 1.0941 & 45 \\
\hline 14 & 1.0945 & 1.0946 & 1.0946 & 1.0949 & 1.0949 & 1.0951 & 1.0951 & 1.0951 & 1.0951 & 1.0951 & 1.0951 & 1.0950 & 60 \\
\hline 15 & 1.1102 & 1.1102 & 1.1102 & 1.1103 & 1.1103 & 1.1104 & 1.1104 & 1.1104 & 1.1104 & 1.1104 & 1.1104 & 1.1104 & 75 \\
\hline
\end{tabular}

The yellow numbers are the maximal $C_{f}$ for identified ply orientations of shared layers.

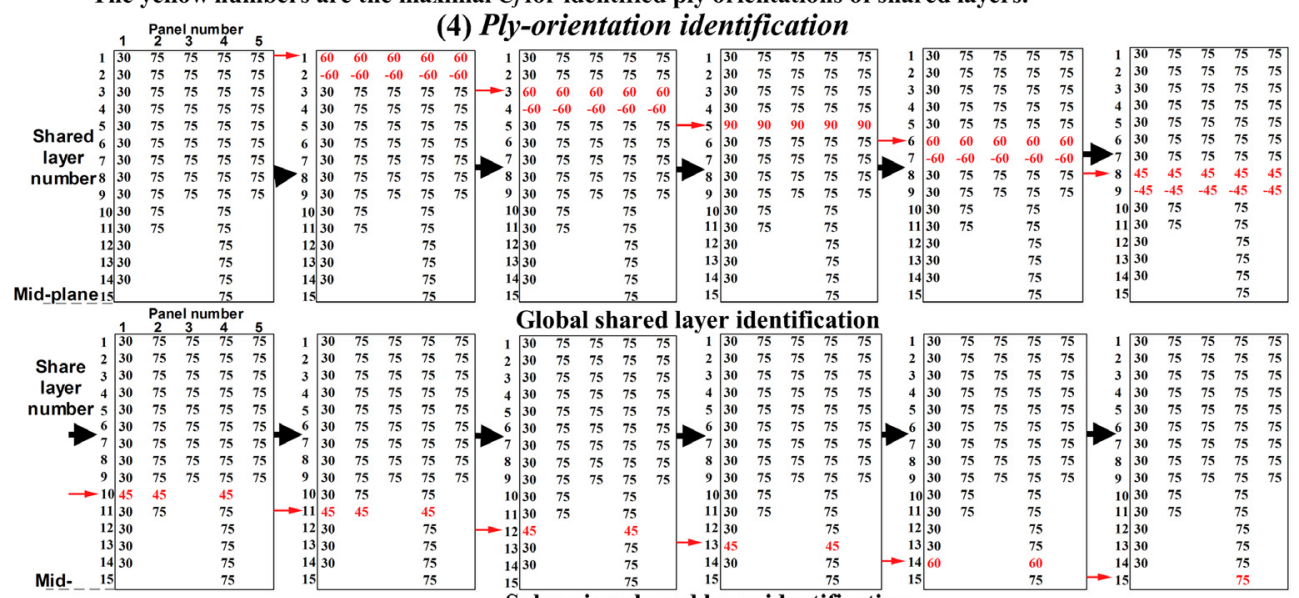

Subregion shared layer identification

Ply orientations are identified from the top to the mid-plane according to the area of shared layers (from large to small), after identification, reset the ply orientations to the best ply orientation of each panel.

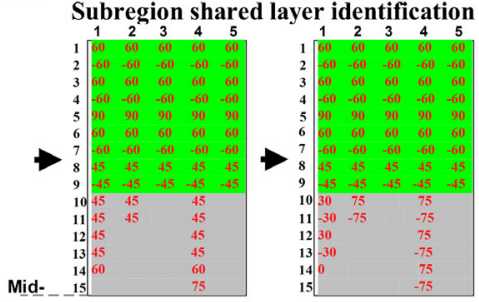

Adjust the ply orientations to satisfy balance constraint (5) Demonstration of ply-orientation identification

Fig. 12. Design process of the 5-panel example: (a) from section 3.1 to 3.4 ; (b) from section 3.5 to 3.8 . 


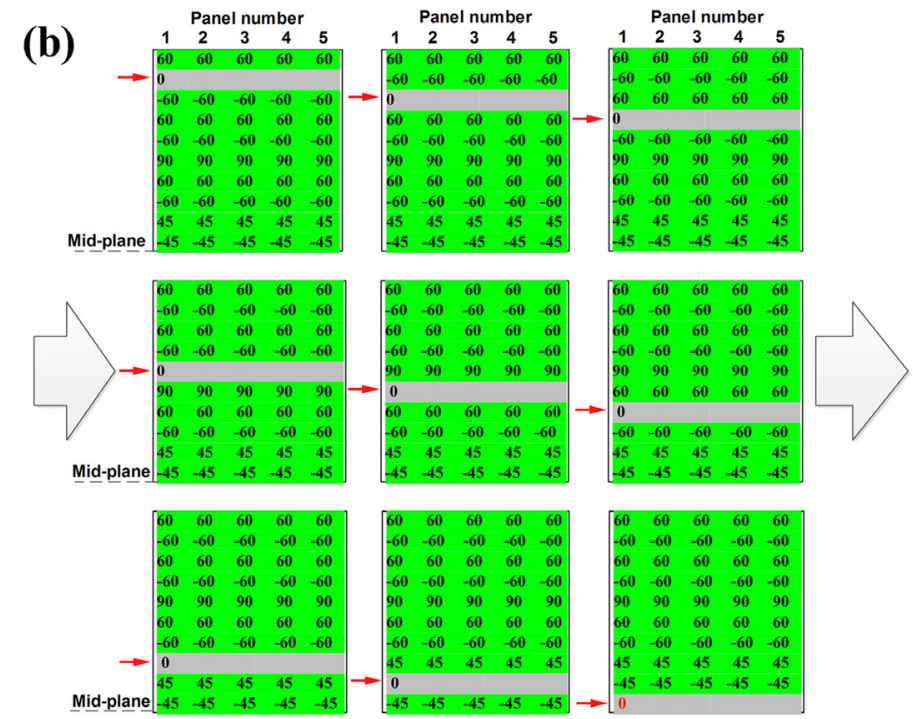

Insert the subregion shared layer from the top to the mid-plane.

Choose the one does not violate any constraint with maximal $C_{\text {ave }}$. Save the sequence for insert operation of next subregion shared layer. In this example, the last case is selected for further operation.
Insert subregion shared layers into the global shared layers

\begin{tabular}{cccccc}
\hline $\begin{array}{c}\text { Insert } \\
\text { number }\end{array}$ & \multicolumn{5}{c}{ Stacking sequence } \\
\hline & 1 & 2 & 3 & 4 & 5 \\
11 & 60 & 60 & 60 & 60 & 60 \\
10 & & & & -75 & \\
9 & & -75 & & -75 & \\
8 & & & & 75 & \\
7 & & & & 75 & \\
3 & 30 & & & & \\
2 & 30 & & & & \\
& 60 & 60 & 60 & 60 & 60 \\
& 60 & 60 & 60 & 60 & 60 \\
& 45 & 45 & 45 & 45 & 45 \\
& 90 & 90 & 90 & 90 & 90 \\
& -60 & -60 & -60 & -60 & -60 \\
6 & & 75 & & 75 & \\
5 & -30 & & & & \\
4 & -30 & & & & \\
& -60 & -60 & -60 & -60 & -60 \\
& -60 & -60 & -60 & -60 & -60 \\
1 & -45 & -45 & -45 & -45 & -45 \\
\hline
\end{tabular}

The green rows are global shared layers. All subregion shared layers are inserted, obtain the optimal sequence.

(6) Demonstration of insert layer operation for sequence optimization

(7) Sequence optimization

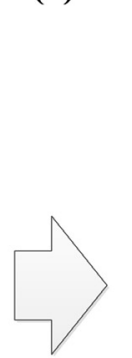

Mid-plane
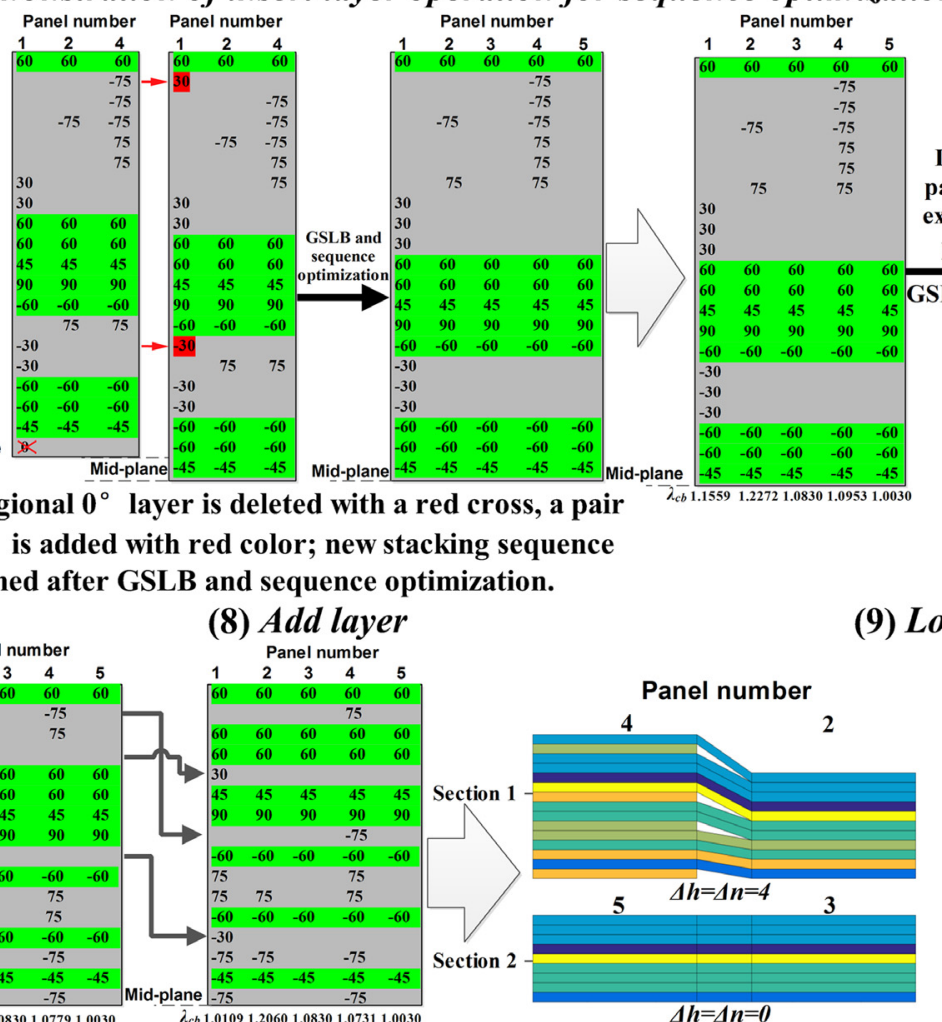

(9) Local blending

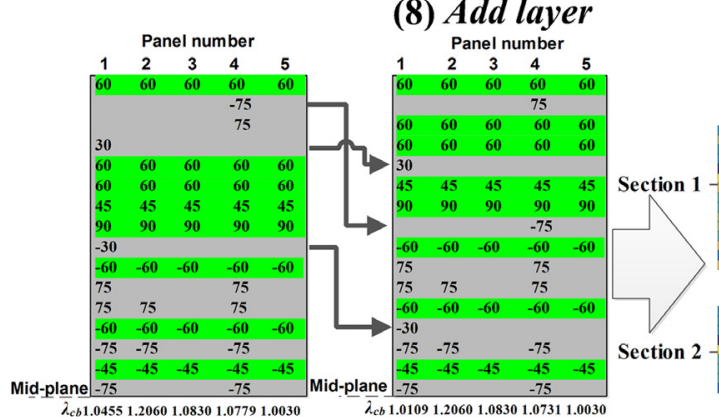

Three single layers: $-75^{\circ}, 30^{\circ}$ and $75^{\circ}$ are re-arranged between large shared layers to decrease the continuity ply drops.

\section{(10) Sequence adjustment}

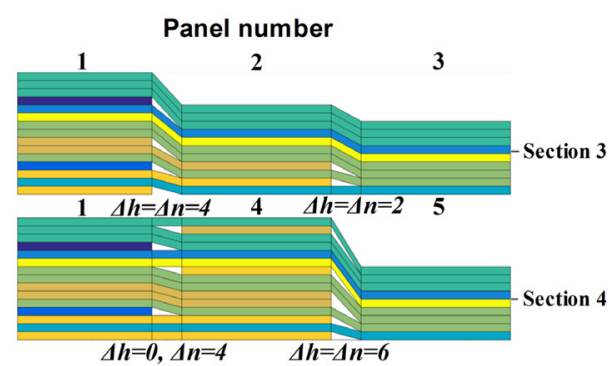

Thickness difference $\Delta h$ and ply drop numbers $\Delta n$ of adjacent zones are calculated. At all adjacent zones, the number of ply drops $\Delta n$ is equal to the thickness difference $\Delta h$ except for the zones between panels $\{1,4\}$, since their best ply orientations are different, and their ply orientations are limited by their bucking load factors. The total weight of the optimal solution is $6.6085 \mathrm{~kg}$.

\section{(11) Sections of optimal restults}

Fig. 12 (continued) 


\section{Algorithm 9: Sequence adjustment}

(1) Assume that 'share_num' is the number of shared layers of the Optimal_sequence, and use the array 'covered_panel' to record the panels covered by a shared layer.

For $i=1$ : share_num

Delete shared layer $(i)$ from its original position, ensure the sequence of panels

covered by the shared layer does not violate any

constraints.

Save the sequence as Origional_sequence.

For $k=i+1$ : share_num

Insert shared layer $(i)$ to stacking position $k$.

For $j=1$ : length $($ shared layer $(i))$

Ensure the sequence of covered_panel $(j)$ does not

violate any constraint;

Evaluate $\lambda_{c b}$ of covered_panel $(j)$;

Ensure $\lambda_{c b} \geqslant 1$;

Evaluate the continuity ply-drop numbers of shared

layer $(i)$ at the insert

position $k$;

Save the $\lambda_{c b}$, continuity ply-drop numbers of

covered_panel $(j)$ and the

sequence.

End For

Reset the sequence to Origional_sequence.

End For

Choose the sequence with minimal ply-drop numbers, save this sequence as

Origional_sequence for insert operation of the next shared layer.

\section{End For}

Finally, after the sequence adjustment, the Optimal_sequence with minimal continuity

ply drops is obtained.

For the 5-panel example, the design process is shown in Fig. 12 (a) and (b) with detailed explanation of each technique introduced above.

\section{Numerical experiments}

A benchmark problem consists of 18 panels previously studied by various researchers $[4-11,15]$ is adopted to verify the proposed design framework, as shown in Fig. 13(a). The definition of 10 sections of the 18 panels is shown in Fig. 13(b). Twelve candidate ply orientations are set as $\left\{0^{\circ}, \pm 15^{\circ}, \pm 30^{\circ}, \pm 45^{\circ}, \pm 60^{\circ}, \pm 75^{\circ}, 90^{\circ}\right\}$, legend of these ply orientations is shown in Fig. 13(c). The adjacent matrix [24] of connectivity relationship for the 18 panels is:

$A=\left[\begin{array}{llllllllllllllllll}0 & 1 & 0 & 0 & 0 & 0 & 0 & 0 & 1 & 0 & 0 & 0 & 0 & 0 & 0 & 0 & 0 & 0 \\ 1 & 0 & 1 & 0 & 0 & 1 & 0 & 0 & 0 & 1 & 0 & 0 & 0 & 0 & 0 & 0 & 0 & 0 \\ 0 & 1 & 0 & 1 & 0 & 1 & 0 & 0 & 0 & 0 & 0 & 0 & 0 & 0 & 0 & 0 & 0 & 0 \\ 0 & 0 & 1 & 0 & 1 & 0 & 1 & 0 & 0 & 0 & 0 & 0 & 0 & 0 & 0 & 0 & 0 & 0 \\ 0 & 0 & 0 & 1 & 0 & 0 & 0 & 1 & 0 & 0 & 0 & 0 & 0 & 0 & 0 & 0 & 0 & 0 \\ 0 & 1 & 1 & 0 & 0 & 0 & 1 & 0 & 0 & 0 & 0 & 0 & 0 & 0 & 0 & 0 & 0 & 0 \\ 0 & 0 & 0 & 1 & 0 & 1 & 0 & 1 & 0 & 0 & 0 & 0 & 0 & 0 & 0 & 0 & 0 & 0 \\ 0 & 0 & 0 & 0 & 1 & 0 & 1 & 0 & 0 & 0 & 0 & 0 & 0 & 0 & 0 & 0 & 0 & 0 \\ 1 & 0 & 0 & 0 & 0 & 0 & 0 & 0 & 0 & 1 & 1 & 0 & 0 & 0 & 0 & 0 & 0 & 0 \\ 0 & 1 & 0 & 0 & 0 & 0 & 0 & 0 & 1 & 0 & 0 & 1 & 0 & 0 & 0 & 0 & 0 & 0 \\ 0 & 0 & 0 & 0 & 0 & 0 & 0 & 0 & 1 & 0 & 0 & 1 & 0 & 0 & 0 & 0 & 0 & 0 \\ 0 & 0 & 0 & 0 & 0 & 0 & 0 & 0 & 0 & 1 & 1 & 0 & 1 & 0 & 0 & 1 & 0 & 0 \\ 0 & 0 & 0 & 0 & 0 & 0 & 0 & 0 & 0 & 0 & 0 & 1 & 0 & 1 & 0 & 1 & 0 & 0 \\ 0 & 0 & 0 & 0 & 0 & 0 & 0 & 0 & 0 & 0 & 0 & 0 & 1 & 0 & 1 & 0 & 1 & 0 \\ 0 & 0 & 0 & 0 & 0 & 0 & 0 & 0 & 0 & 0 & 0 & 0 & 0 & 1 & 0 & 0 & 0 & 1 \\ 0 & 0 & 0 & 0 & 0 & 0 & 0 & 0 & 0 & 0 & 0 & 1 & 1 & 0 & 0 & 0 & 1 & 0 \\ 0 & 0 & 0 & 0 & 0 & 0 & 0 & 0 & 0 & 0 & 0 & 0 & 0 & 1 & 0 & 1 & 0 & 1 \\ 0 & 0 & 0 & 0 & 0 & 0 & 0 & 0 & 0 & 0 & 0 & 0 & 0 & 0 & 1 & 0 & 1 & 0\end{array}\right]$

The material properties are the same with the case of the 5-panel example in Section 3. The objective function is Eq. (8), the design process will be executed step by step, with important intermediate results presented after each step. The constraints in Eq. (8) are divided and enforced in each step. All codes are made in Matlab R2014b. The execution time for finding the best solution is around $13 \mathrm{~s}$ using the Intel Core i7-4800MQ $(2.7 \mathrm{GHz})$ processor. The optimal results are compared with previous studies.

Based on the flowchart in Fig. 4, first, Algorithm 1 is employed to evaluate the $\lambda_{c b}$ of a single lamina, results are sorted in Table 4 from the maximal to the minimal with their corresponding ply orientations re-written in MSPT of Table 5: the best ply orientation of panels $1,2,9,10,11$ and 12 is $\pm 30^{\circ}$, while the best ply orientation of other panels is $\pm 75^{\circ}$. Second, Algorithm 2 is used to get the minimum-thickness distribution as shown in Table 6 , the results are the same with that derived from continuous variables of Table A2 given in [10]. Third, Algorithm 3 is employed to obtain the shared layers. They are presented in Table 7, where a row represents a shared layer, the blanks means the panels are not covered by the shared layers. There are 8 global shared layers and 18

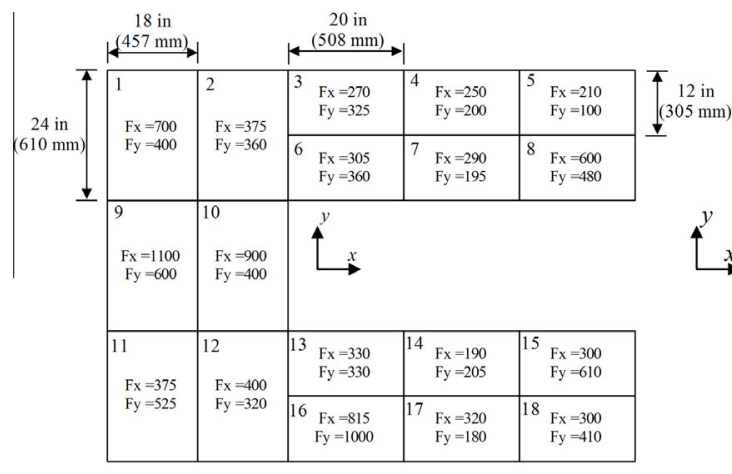

(a)

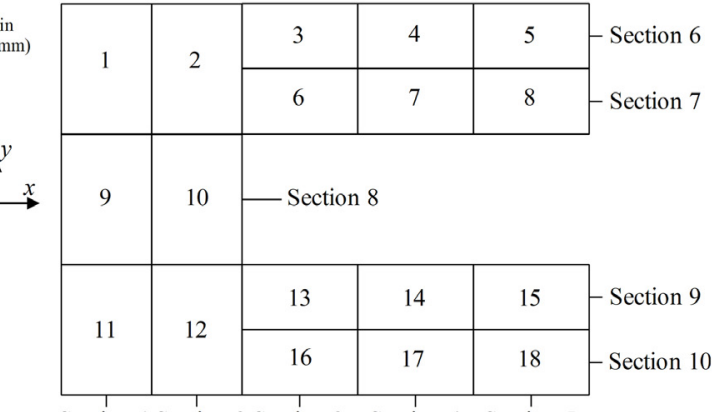

(b)

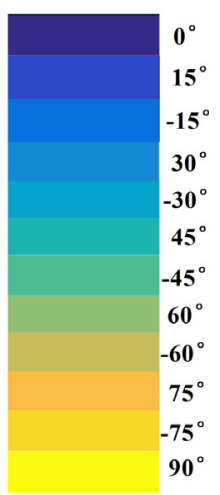

(c)

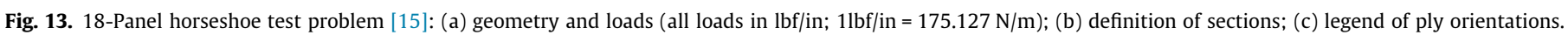


Table 4

Buckling load factors $\left(1 \times 10^{-4}\right)$ for one lamina of the 18 -panel problem.

\begin{tabular}{|c|c|c|c|c|c|c|c|c|c|c|c|c|c|c|c|c|c|c|}
\hline \multirow[t]{2}{*}{ One lamina } & \multicolumn{18}{|c|}{ Ply-orientation contribution factor of panels } \\
\hline & 1 & 2 & 3 & 4 & 5 & 6 & 7 & 8 & 9 & 10 & 11 & 12 & 13 & 14 & 15 & 16 & 17 & 18 \\
\hline$[0]_{s}$ & 2.12 & 3.40 & 2.37 & 3.45 & 5.70 & 2.13 & 3.34 & 1.44 & 1.37 & 1.75 & 2.93 & 3.39 & 2.23 & 3.66 & 1.39 & 0.77 & 3.39 & 1.93 \\
\hline$[15]_{s}$ & 2.26 & 3.62 & 3.43 & 5.00 & 8.25 & 3.08 & 4.84 & 2.08 & 1.46 & 1.86 & 3.12 & 3.61 & 3.23 & 5.30 & 2.02 & 1.12 & 4.91 & 2.80 \\
\hline$[-15]_{s}$ & 2.26 & 3.62 & 3.43 & 5.00 & 8.25 & 3.08 & 4.84 & 2.08 & 1.46 & 1.86 & 3.12 & 3.61 & 3.23 & 5.30 & 2.02 & 1.12 & 4.91 & 2.80 \\
\hline$[30]_{s}$ & 2.48 & 3.98 & 5.93 & 8.64 & 14.26 & 5.33 & 8.36 & 3.60 & 1.60 & 2.04 & 3.43 & 3.96 & 5.58 & 9.16 & 3.49 & 1.94 & 8.48 & 4.83 \\
\hline$[-30]_{S}$ & 2.48 & 3.98 & 5.93 & 8.64 & 14.26 & 5.33 & 8.36 & 3.60 & 1.60 & 2.04 & 3.43 & 3.96 & 5.58 & 9.16 & 3.49 & 1.94 & 8.48 & 4.83 \\
\hline$[45]_{s}$ & 2.40 & 3.84 & 8.40 & 12.23 & 20.19 & 7.55 & 11.84 & 5.09 & 1.54 & 1.97 & 3.31 & 3.83 & 7.90 & 12.97 & 4.94 & 2.74 & 12.01 & 6.85 \\
\hline$[-45]_{s}$ & 2.40 & 3.84 & 8.40 & 12.23 & 20.19 & 7.55 & 11.84 & 5.09 & 1.54 & 1.97 & 3.31 & 3.83 & 7.90 & 12.97 & 4.94 & 2.74 & 12.01 & 6.85 \\
\hline$[60]_{s}$ & 1.87 & 3.00 & 9.77 & 14.22 & 23.49 & 8.78 & 13.78 & 5.93 & 1.20 & 1.54 & 2.58 & 2.99 & 9.19 & 15.09 & 5.74 & 3.19 & 13.97 & 7.96 \\
\hline$[-60]_{s}$ & 1.87 & 3.00 & 9.77 & 14.22 & 23.49 & 8.78 & 13.78 & 5.93 & 1.20 & 1.54 & 2.58 & 2.99 & 9.19 & 15.09 & 5.74 & 3.19 & 13.97 & 7.96 \\
\hline$[75]_{s}$ & 1.20 & 1.93 & 10.08 & 14.67 & 24.23 & 9.06 & 14.21 & 6.11 & 0.78 & 0.99 & 1.66 & 1.92 & 9.48 & 15.57 & 5.93 & 3.29 & 14.42 & 8.22 \\
\hline$[-75]_{s}$ & 1.20 & 1.93 & 10.08 & 14.67 & 24.23 & 9.06 & 14.21 & 6.11 & 0.78 & 0.99 & 1.66 & 1.92 & 9.48 & 15.57 & 5.93 & 3.29 & 14.42 & 8.22 \\
\hline$[90]_{s}$ & 0.90 & 1.44 & 10.05 & 14.63 & 24.15 & 9.03 & 14.17 & 6.09 & 0.58 & 0.74 & 1.24 & 1.44 & 9.45 & 15.51 & 5.91 & 3.28 & 14.37 & 8.19 \\
\hline
\end{tabular}

Table 5

Multi-panel sequential permutation table (MSPT) of the 18-panel problem.

\begin{tabular}{|c|c|c|c|c|c|c|c|c|c|c|c|c|c|c|c|c|c|c|}
\hline \multirow{2}{*}{$\begin{array}{l}\text { Optimal number } \\
\text { of ply orientation }\end{array}$} & \multicolumn{18}{|c|}{ Ply orientations sort from best to worst of panels } \\
\hline & 1 & 2 & 3 & 4 & 5 & 6 & 7 & 8 & 9 & 10 & 11 & 12 & 13 & 14 & 15 & 16 & 17 & 18 \\
\hline 1 & 30 & 30 & 75 & 75 & 75 & 75 & 75 & 75 & 30 & 30 & 30 & 30 & 75 & 75 & 75 & 75 & 75 & 75 \\
\hline 2 & -30 & -30 & -75 & -75 & -75 & -75 & -75 & -75 & -30 & -30 & -30 & -30 & -75 & -75 & -75 & -75 & -75 & -75 \\
\hline 3 & 45 & 45 & 90 & 90 & 90 & 90 & 90 & 90 & 45 & 45 & 45 & 45 & 90 & 90 & 90 & 90 & 90 & 90 \\
\hline 4 & -45 & -45 & 60 & 60 & 60 & 60 & 60 & 60 & -45 & -45 & -45 & -45 & 60 & 60 & 60 & 60 & 60 & 60 \\
\hline 5 & 15 & 15 & -60 & -60 & -60 & -60 & -60 & -60 & 15 & 15 & 15 & 15 & -60 & -60 & -60 & -60 & -60 & -60 \\
\hline 6 & -15 & -15 & 45 & 45 & 45 & 45 & 45 & 45 & -15 & -15 & -15 & -15 & 45 & 45 & 45 & 45 & 45 & 45 \\
\hline 7 & 0 & 0 & -45 & -45 & -45 & -45 & -45 & -45 & 0 & 0 & 0 & 0 & -45 & -45 & -45 & -45 & -45 & -45 \\
\hline 8 & 60 & 60 & 30 & 30 & 30 & 30 & 30 & 30 & 60 & 60 & 60 & 60 & 30 & 30 & 30 & 30 & 30 & 30 \\
\hline 9 & -60 & -60 & -30 & -30 & -30 & -30 & -30 & -30 & -60 & -60 & -60 & -60 & -30 & -30 & -30 & -30 & -30 & -30 \\
\hline 10 & 75 & 75 & 15 & 15 & 15 & 15 & 15 & 15 & 75 & 75 & 75 & 75 & 15 & 15 & 15 & 15 & 15 & 15 \\
\hline 11 & -75 & -75 & -15 & -15 & -15 & -15 & -15 & -15 & -75 & -75 & -75 & -75 & -15 & -15 & -15 & -15 & -15 & -15 \\
\hline 12 & 90 & 90 & 0 & 0 & 0 & 0 & 0 & 0 & 90 & 90 & 90 & 90 & 0 & 0 & 0 & 0 & 0 & 0 \\
\hline
\end{tabular}

The best ply orientation (bold value) is the first row with number 1 , the worst ply-orientation is the last row with number 12 .

subregion shared layers. Fourth, Algorithm 4 is used to identify ply orientations of shared layers (see Tables 8 and 9), followed by Algorithm 5 to adjust the number of ply orientations of each panel to satisfy the balance and disorientation requirements. Ply orientations of shared layers in Table 7 are identified in Table 8, the highlighted yellow positions in every row are the best ply orientations with the maximal contribution factors $C_{f}$ (Eq. (9)) except for the fourth row. To satisfy the disorientation constraint, $0^{\circ}$ or $90^{\circ}$ layer should be set in the sequence. As shown in Table 9, two pairs of $\pm 45^{\circ}$, a pair of $\pm 60^{\circ}$ and two $90^{\circ}$ ply orientations are identified

Table 6

Minimum-thickness distribution for the 18-panel problem.

\begin{tabular}{rlrl}
\hline Panel number & $\begin{array}{l}\text { Stacking } \\
\text { sequence }\end{array}$ & $\begin{array}{l}\text { Minimum thickness } \\
\text { in half laminate }\end{array}$ & $\lambda_{c b}$ \\
\hline 1 & {$\left[( \pm 30)_{8}\right]_{s}$} & 16 & 1.0172 \\
2 & {$\left[( \pm 30)_{7}\right]_{s}$} & 14 & 1.0914 \\
3 & {$\left[( \pm 75)_{5}\right]_{s}$} & 10 & 1.0079 \\
4 & {$\left[( \pm 75)_{4} / 90\right]_{s}$} & 9 & 1.0697 \\
5 & {$\left[( \pm 75)_{4}\right]_{s}$} & 8 & 1.2408 \\
6 & {$\left[( \pm 75)_{5} / 90\right]_{s}$} & 11 & 1.2056 \\
7 & {$\left[( \pm 75)_{4} / 90\right]_{s}$} & 9 & 1.0362 \\
8 & {$\left[( \pm 75)_{6}\right]_{s}$} & 12 & 1.0565 \\
9 & {$\left[( \pm 30)_{9} / 0\right]_{s}$} & 19 & 1.0960 \\
10 & {$\left[( \pm 30)_{8} / 0\right]_{s}$} & 17 & 1.0031 \\
11 & {$\left[( \pm 30)_{7} / 0\right]_{s}$} & 15 & 1.1565 \\
12 & {$\left[( \pm 30)_{7}\right]_{s}$} & 14 & 1.0867 \\
13 & {$\left[( \pm 75)_{5} / 90\right]_{s}$} & 11 & 1.2620 \\
14 & $\left.\left[( \pm 75)_{4} / 90\right]\right]_{s}$ & 9 & 1.1347 \\
15 & {$\left[( \pm 75)_{6}\right]_{s}$} & 12 & 1.0242 \\
16 & $\left.\left[( \pm 75)_{7} / 90\right]\right]_{s}$ & 15 & 1.1104 \\
17 & {$\left[( \pm 75)_{4} / 90\right]_{s}$} & 9 & 1.0509 \\
18 & {$\left[( \pm 75)_{5} / 90\right]_{s}$} & 11 & 1.0934 \\
\hline
\end{tabular}

for 8 global shared layers, while other subregion shared layers are preferable to choose their best ply orientations $30^{\circ}$ or $75^{\circ}$. At the next stage, the detailed changing procedures for number of ply orientations and stacking sequences are demonstrated in Figs. 14 and 15, respectively.

Fig. 14(a) provides detailed number of ply orientations in Table 9. The adjusted results of Algorithm 5 are presented in Fig. 14(b), where the balance requirement is satisfied. Then, the GSLB method is used to recalculate the shared-layer's shape, as shown in Table 10, where the shared-layer's number is written as $\operatorname{SL} i(i=1,2,3 \ldots)$ and the shared layers of various ply orientations are listed from maximal to minimal. Fifth, Algorithm 6 is implemented to optimize the sequence using shared layers in Table 10, the obtained sequence is shown in Fig. 15(a). The rows highlighted in green are the global shared layers and set as an initial frame sequence (there are many initial frame sequences, the one presented in Fig. 15(a) corresponds to the best solution). Then the subregion shared layers in Table 10 are inserted into it one by one from the outermost to the mid-plane position without violation of individual panel constraints. The insert number of shared layers is listed at the first column of Fig. 15(a), $\lambda_{c b}$ of these panels are calculated and presented in the first row of Table 11: panels $1,3,4,7,10,15$ and 17 violate the buckling constraint. Sixth, Algorithm 7 is employed to add layers to the panels $1,3,4,7,10,15$ and 17. The add layer procedure is shown in Fig. 15(b): one $0^{\circ}$ or $90^{\circ}$ layer is added to each panel of 1,3 and 15 , one $0^{\circ}$ or $90^{\circ}$ layer is deleted from each panel of $4,7,10$ and 17 by adding a pair of their best ply orientations $\pm 30^{\circ}$ or $\pm 75^{\circ}$ and blended with original shared layers. Deleted layers are labeled with a red cross and added layers are highlighted with orange. Number of ply orientations after 
adding layers are provided in Fig. 14(c). The situation that one added layer blend with two separated layers is appeared at panel 17 (Fig. 15 (b)), a pair of $\pm 75^{\circ}$ blend with two separated shared layers $\{13,16\}$ and $\{15,18\}$. Thus, the GSLB method and sequenceoptimization strategy are employed to optimize the structure again, stacking sequence results are presented in Fig. 15(c) with corresponding layers in Fig. 16(a), (b) and sections in Fig. 17 (a), (c). $\lambda_{c b}$ of Fig. 15(c) are presented at the second row of Table 11; some $\lambda_{c b}$ have at least a $20 \%$ margin $\left(\lambda_{c b}>1.2\right)$, e.g. panels $3,4,7,15$ and 17. In these panels, the blending property can be improved by adjusting the ply-orientation numbers with a decrease of their $\lambda_{c b}$. Thus, the local blending algorithm (Algorithm 8) is implemented, as shown in shaded parts of Fig. 14(c) and (d). Compared to the sequence in Fig. 14(c), a pair of $\pm 75^{\circ}$ plies is exchanged to $\pm 30^{\circ}$ plies in panels 3, 4, 6, 7, 13, 15, 16 and 17 in Fig. 14(d). The gray parts in Fig. 15(c) and (d) demonstrate the exchanging process; the new $\pm 30^{\circ}$ layers are blended to bigger shared layers in panels 3, 4, 6, 7, 13, 16 and 17 (see Figs. 16 and 17) except for panel 15. Though the changed $\pm 30^{\circ}$ layers in panel 15 is a single layer, the original single layers $\pm 75^{\circ}$ (above the highlighted with gray $\pm 75^{\circ}$ layers in Fig. 15(c)) are blended to the layers of panel 18 (see Fig. 15(d)). Since the numbers of ply orientations are adjusted, the GSLB method and sequence optimization strategy are implemented again; the results are presented in Fig. 15(d). To decrease

Table 7

Results 1 of global shared-layer blending method for the 18-panel problem.

\begin{tabular}{|c|c|c|c|c|c|c|c|c|c|c|c|c|c|c|c|c|c|c|c|}
\hline \multirow[t]{2}{*}{ Shared layer type } & \multirow[t]{2}{*}{ Shared layer number } & \multicolumn{18}{|c|}{ Panels covered by shared layers } \\
\hline & & 1 & 2 & 3 & 4 & 5 & 6 & 7 & 8 & 9 & 10 & 11 & 12 & 13 & 14 & 15 & 16 & 17 & 18 \\
\hline \multirow[t]{8}{*}{ Global shared layers } & 1 & 1 & 2 & 3 & 4 & 5 & 6 & 7 & 8 & 9 & 10 & 11 & 12 & 13 & 14 & 15 & 16 & 17 & 18 \\
\hline & 2 & 1 & 2 & 3 & 4 & 5 & 6 & 7 & 8 & 9 & 10 & 11 & 12 & 13 & 14 & 15 & 16 & 17 & 18 \\
\hline & 3 & 1 & 2 & 3 & 4 & 5 & 6 & 7 & 8 & 9 & 10 & 11 & 12 & 13 & 14 & 15 & 16 & 17 & 18 \\
\hline & 4 & 1 & 2 & 3 & 4 & 5 & 6 & 7 & 8 & 9 & 10 & 11 & 12 & 13 & 14 & 15 & 16 & 17 & 18 \\
\hline & 5 & 1 & 2 & 3 & 4 & 5 & 6 & 7 & 8 & 9 & 10 & 11 & 12 & 13 & 14 & 15 & 16 & 17 & 18 \\
\hline & 6 & 1 & 2 & 3 & 4 & 5 & 6 & 7 & 8 & 9 & 10 & 11 & 12 & 13 & 14 & 15 & 16 & 17 & 18 \\
\hline & 7 & 1 & 2 & 3 & 4 & 5 & 6 & 7 & 8 & 9 & 10 & 11 & 12 & 13 & 14 & 15 & 16 & 17 & 18 \\
\hline & 8 & 1 & 2 & 3 & 4 & 5 & 6 & 7 & 8 & 9 & 10 & 11 & 12 & 13 & 14 & 15 & 16 & 17 & 18 \\
\hline \multirow[t]{18}{*}{ Subregion shared layers } & 9 & 1 & 2 & 3 & 4 & & 6 & 7 & 8 & 9 & 10 & 11 & 12 & 13 & 14 & 15 & 16 & 17 & 18 \\
\hline & 10 & 1 & 2 & 3 & & & 6 & & & 9 & 10 & 11 & 12 & 13 & & & 16 & & \\
\hline & 11 & 1 & 2 & & & & 6 & & & 9 & 10 & 11 & 12 & 13 & & & 16 & & \\
\hline & 12 & 1 & 2 & & & & & & & 9 & 10 & 11 & 12 & & & & 16 & & \\
\hline & 13 & 1 & 2 & & & & & & & 9 & 10 & 11 & 12 & & & & 16 & & \\
\hline & 14 & 1 & 2 & & & & & & & 9 & 10 & 11 & 12 & & & & 16 & & \\
\hline & 15 & 1 & & & & & & & & 9 & 10 & 11 & & & & & & & \\
\hline & 16 & 1 & & & & & & & & 9 & 10 & & & & & & & & \\
\hline & 17 & & & & & & & & & & & & & & & 15 & & & 18 \\
\hline & 18 & & & & & & & & & & & & & & & 15 & & & 18 \\
\hline & 19 & & & & & & & & & 9 & 10 & & & & & & & & \\
\hline & 20 & & & & & & & & 8 & & & & & & & & & & \\
\hline & 21 & & & & & & & & 8 & & & & & & & & & & \\
\hline & 22 & & & & & & & & 8 & & & & & & & & & & \\
\hline & 23 & & & & & & & & & & & & & & & 15 & & & \\
\hline & 24 & & & & & & & & & & & & & & & & 16 & & \\
\hline & 25 & & & & & & & & & 9 & & & & & & & & & \\
\hline & 26 & & & & & & & & & 9 & & & & & & & & & \\
\hline
\end{tabular}

Table 8

Ply-orientation identification process for the 18-panel problem.

\begin{tabular}{|c|c|c|c|c|c|c|c|c|c|c|c|c|c|}
\hline \multirow{2}{*}{$\begin{array}{c}\text { Shared } \\
\text { layer } \\
\text { number }\end{array}$} & 1 & 2 & 3 & 4 & 5 & 6 & 7 & 8 & 9 & 10 & 11 & 12 & \multirow{2}{*}{$\begin{array}{c}\text { Ply } \\
\text { orientation }\end{array}$} \\
\hline & $0^{\circ}$ & $15^{\circ}$ & $-15^{\circ}$ & $30^{\circ}$ & $-30^{\circ}$ & $45^{\circ}$ & $-45^{\circ}$ & $60^{\circ}$ & $-60^{\circ}$ & $75^{\circ}$ & $-75^{\circ}$ & $90^{\circ}$ & \\
\hline 1,2 & 0.9605 & 0.9820 & 0.9820 & 1.0286 & 1.0286 & 1.0634 & 1.0634 & 1.0650 & 1.0650 & 1.0451 & 1.0451 & 1.0334 & $60,-60$ \\
\hline 3,4 & 1.0085 & 1.0226 & 1.0226 & 1.0526 & 1.0526 & 1.0739 & 1.0739 & 1.0724 & 1.0724 & 1.0569 & 1.0569 & 1.0481 & $45,-45$ \\
\hline 5,6 & 1.0451 & 1.0534 & 1.0534 & 1.0708 & 1.0708 & 1.0819 & 1.0819 & 1.0786 & 1.0786 & 1.0669 & 1.0669 & 1.0607 & $45,-45$ \\
\hline 7,8 & 1.0704 & 1.0746 & 1.0746 & 1.0831 & 1.0831 & 1.0875 & 1.0875 & 1.0835 & 1.0835 & 1.0753 & 1.0753 & 1.0712 & 90,90 \\
\hline 9 & 1.0772 & 1.0791 & 1.0791 & 1.0827 & 1.0827 & 1.0836 & 1.0836 & 1.0801 & 1.0801 & 1.0746 & 1.0746 & 1.0720 & 45 \\
\hline 10 & 1.0889 & 1.0905 & 1.0905 & 1.0932 & 1.0932 & 1.0932 & 1.0932 & 1.0887 & 1.0887 & 1.0826 & 1.0826 & 1.0798 & 30 \\
\hline 11 & 1.0970 & 1.0981 & 1.0981 & 1.1001 & 1.1001 & 1.0999 & 1.0999 & 1.0964 & 1.0964 & 1.0918 & 1.0918 & 1.0896 & 30 \\
\hline 12 & 1.0754 & 1.0763 & 1.0763 & 1.0777 & 1.0777 & 1.0775 & 1.0775 & 1.0748 & 1.0748 & 1.0712 & 1.0712 & 1.0696 & 30 \\
\hline 13 & 1.0766 & 1.0771 & 1.0771 & 1.0780 & 1.0780 & 1.0778 & 1.0778 & 1.0761 & 1.0761 & 1.0739 & 1.0739 & 1.0729 & 30 \\
\hline 14 & 1.0774 & 1.0776 & 1.0776 & 1.0781 & 1.0781 & 1.0780 & 1.0780 & 1.0770 & 1.0770 & 1.0758 & 1.0758 & 1.0752 & 30 \\
\hline 15 & 1.0677 & 1.0679 & 1.0679 & 1.0682 & 1.0682 & 1.0681 & 1.0681 & 1.0673 & 1.0673 & 1.0662 & 1.0662 & 1.0657 & 30 \\
\hline 16 & 1.0384 & 1.0386 & 1.0386 & 1.0388 & 1.0388 & 1.0387 & 1.0387 & 1.0382 & 1.0382 & 1.0375 & 1.0375 & 1.0372 & 30 \\
\hline 17 & 1.0523 & 1.0532 & 1.0532 & 1.0553 & 1.0553 & 1.0574 & 1.0574 & 1.0585 & 1.0585 & 1.0588 & 1.0588 & 1.0588 & 75 \\
\hline 18 & 1.0569 & 1.0572 & 1.0572 & 1.0578 & 1.0578 & 1.0584 & 1.0584 & 1.0587 & 1.0587 & 1.0588 & 1.0588 & 1.0588 & 75 \\
\hline 19 & 1.0494 & 1.0494 & 1.0494 & 1.0496 & 1.0496 & 1.0495 & 1.0495 & 1.0492 & 1.0492 & 1.0488 & 1.0488 & 1.0486 & 30 \\
\hline 20 & 1.0477 & 1.0489 & 1.0489 & 1.0518 & 1.0518 & 1.0546 & 1.0546 & 1.0562 & 1.0562 & 1.0565 & 1.0565 & 1.0565 & 75 \\
\hline 21 & 1.0533 & 1.0537 & 1.0537 & 1.0548 & 1.0548 & 1.0558 & 1.0558 & 1.0564 & 1.0564 & 1.0565 & 1.0565 & 1.0565 & 75 \\
\hline 22 & 1.0561 & 1.0561 & 1.0561 & 1.0563 & 1.0563 & 1.0564 & 1.0564 & 1.0565 & 1.0565 & 1.0565 & 1.0565 & 1.0565 & 75 \\
\hline 23 & 1.0237 & 1.0238 & 1.0238 & 1.0239 & 1.0239 & 1.0241 & 1.0241 & 1.0241 & 1.0241 & 1.0242 & 1.0242 & 1.0242 & 75 \\
\hline 24 & 1.1102 & 1.1102 & 1.1102 & 1.1103 & 1.1103 & 1.1104 & 1.1104 & 1.1104 & 1.1104 & 1.1104 & 1.1104 & 1.1104 & 75 \\
\hline 25 & 1.0959 & 1.0959 & 1.0959 & 1.0960 & 1.0960 & 1.0960 & 1.0960 & 1.0958 & 1.0958 & 1.0955 & 1.0955 & 1.0953 & 75 \\
\hline 26 & 1.0960 & 1.0960 & 1.0960 & 1.0960 & 1.0960 & 1.0960 & 1.0960 & 1.0960 & 1.0960 & 1.0960 & 1.0960 & 1.0959 & 75 \\
\hline
\end{tabular}


Table 9

Ply-orientation identification for shared layers of the 18-panel problem.

\begin{tabular}{|c|c|c|c|c|c|c|c|c|c|c|c|c|c|c|c|c|c|c|c|}
\hline \multirow[t]{2}{*}{ Shared-layer type } & \multirow{2}{*}{$\begin{array}{l}\text { Shared-layers } \\
\text { number }\end{array}$} & \multicolumn{18}{|c|}{ The identified ply orientation of shared layers } \\
\hline & & 1 & 2 & 3 & 4 & 5 & 6 & 7 & 8 & 9 & 10 & 11 & 12 & 13 & 14 & 15 & 16 & 17 & 18 \\
\hline \multirow{8}{*}{$\begin{array}{l}\text { Global shared } \\
\text { layers }\end{array}$} & 1 & 60 & 60 & 60 & 60 & 60 & 60 & 60 & 60 & 60 & 60 & 60 & 60 & 60 & 60 & 60 & 60 & 60 & 60 \\
\hline & 2 & -60 & -60 & -60 & -60 & -60 & -60 & -60 & -60 & -60 & -60 & -60 & -60 & -60 & -60 & -60 & -60 & -60 & -60 \\
\hline & 3 & 45 & 45 & 45 & 45 & 45 & 45 & 45 & 45 & 45 & 45 & 45 & 45 & 45 & 45 & 45 & 45 & 45 & 45 \\
\hline & 4 & -45 & -45 & -45 & -45 & -45 & -45 & -45 & -45 & -45 & -45 & -45 & -45 & -45 & -45 & -45 & -45 & -45 & -45 \\
\hline & 5 & 45 & 45 & 45 & 45 & 45 & 45 & 45 & 45 & 45 & 45 & 45 & 45 & 45 & 45 & 45 & 45 & 45 & 45 \\
\hline & 6 & -45 & -45 & -45 & -45 & -45 & -45 & -45 & -45 & -45 & -45 & -45 & -45 & -45 & -45 & -45 & -45 & -45 & -45 \\
\hline & 7 & 90 & 90 & 90 & 90 & 90 & 90 & 90 & 90 & 90 & 90 & 90 & 90 & 90 & 90 & 90 & 90 & 90 & 90 \\
\hline & 8 & 90 & 90 & 90 & 90 & 90 & 90 & 90 & 90 & 90 & 90 & 90 & 90 & 90 & 90 & 90 & 90 & 90 & 90 \\
\hline \multirow{18}{*}{$\begin{array}{l}\text { Subregion shared } \\
\text { layers }\end{array}$} & 9 & 45 & 45 & 45 & 45 & & 45 & 45 & 45 & 45 & 45 & 45 & 45 & 45 & 45 & 45 & 45 & 45 & 45 \\
\hline & 10 & 30 & 30 & 30 & & & 30 & & & 30 & 30 & 30 & 30 & 30 & & & 30 & & \\
\hline & 11 & 30 & 30 & & & & 30 & & & 30 & 30 & 30 & 30 & 30 & & & 30 & & \\
\hline & 12 & 30 & 30 & & & & & & & 30 & 30 & 30 & 30 & & & & 30 & & \\
\hline & 13 & 30 & 30 & & & & & & & 30 & 30 & 30 & 30 & & & & 30 & & \\
\hline & 14 & 30 & 30 & & & & & & & 30 & 30 & 30 & 30 & & & & 30 & & \\
\hline & 15 & 30 & & & & & & & & 30 & 30 & 30 & & & & & & & \\
\hline & 16 & 30 & & & & & & & & 30 & 30 & & & & & & & & \\
\hline & 17 & & & & & & & & & & & & & & & 75 & & & 75 \\
\hline & 18 & & & & & & & & & & & & & & & 75 & & & 75 \\
\hline & 19 & & & & & & & & & 30 & 30 & & & & & & & & \\
\hline & 20 & & & & & & & & 75 & & & & & & & & & & \\
\hline & 21 & & & & & & & & 75 & & & & & & & & & & \\
\hline & 22 & & & & & & & & 75 & & & & & & & & & & \\
\hline & 23 & & & & & & & & & & & & & & & 75 & & & \\
\hline & 24 & & & & & & & & & & & & & & & & 75 & & \\
\hline & 25 & & & & & & & & & 30 & & & & & & & & & \\
\hline & 26 & & & & & & & & & 30 & & & & & & & & & \\
\hline
\end{tabular}

(a)

\begin{tabular}{lcccccccccccc}
\hline Panel & \multicolumn{11}{c}{ Number of ply orientations } \\
\cline { 2 - 5 } & 0 & 15 & -15 & 30 & -30 & 45 & -45 & 60 & -60 & 75 & -75 & 90 \\
\hline 1 & 0 & 0 & 0 & 7 & 0 & 3 & 2 & 1 & 1 & 0 & 0 & 2 \\
2 & 0 & 0 & 0 & 5 & 0 & 3 & 2 & 1 & 1 & 0 & 0 & 2 \\
3 & 0 & 0 & 0 & 1 & 0 & 3 & 2 & 1 & 1 & 0 & 0 & 2 \\
4 & 0 & 0 & 0 & 0 & 0 & 3 & 2 & 1 & 1 & 0 & 0 & 2 \\
5 & 0 & 0 & 0 & 0 & 0 & 2 & 2 & 1 & 1 & 0 & 0 & 2 \\
6 & 0 & 0 & 0 & 2 & 0 & 3 & 2 & 1 & 1 & 0 & 0 & 2 \\
7 & 0 & 0 & 0 & 0 & 0 & 3 & 2 & 1 & 1 & 0 & 0 & 2 \\
8 & 0 & 0 & 0 & 0 & 0 & 3 & 2 & 1 & 1 & 3 & 0 & 2 \\
9 & 0 & 0 & 0 & 10 & 0 & 3 & 2 & 1 & 1 & 0 & 0 & 2 \\
10 & 0 & 0 & 0 & 8 & 0 & 3 & 2 & 1 & 1 & 0 & 0 & 2 \\
11 & 0 & 0 & 0 & 6 & 0 & 3 & 2 & 1 & 1 & 0 & 0 & 2 \\
12 & 0 & 0 & 0 & 5 & 0 & 3 & 2 & 1 & 1 & 0 & 0 & 2 \\
13 & 0 & 0 & 0 & 2 & 0 & 3 & 2 & 1 & 1 & 0 & 0 & 2 \\
14 & 0 & 0 & 0 & 0 & 0 & 3 & 2 & 1 & 1 & 0 & 0 & 2 \\
15 & 0 & 0 & 0 & 0 & 0 & 3 & 2 & 1 & 1 & 3 & 0 & 2 \\
16 & 0 & 0 & 0 & 5 & 0 & 3 & 2 & 1 & 1 & 1 & 0 & 2 \\
17 & 0 & 0 & 0 & 0 & 0 & 3 & 2 & 1 & 1 & 0 & 0 & 2 \\
18 & 0 & 0 & 0 & 0 & 0 & 3 & 2 & 1 & 1 & 2 & 0 & 2 \\
\hline
\end{tabular}

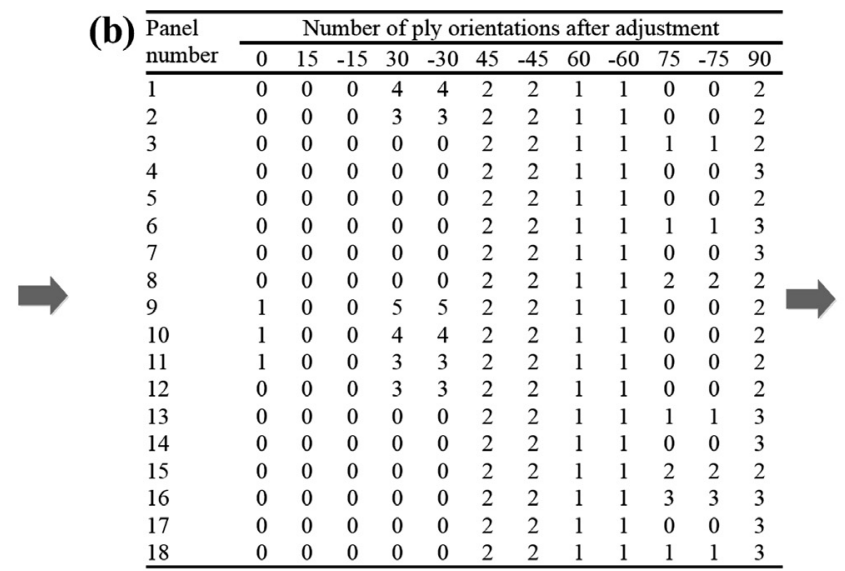

(c) \begin{tabular}{lcccccccccccccc}
\hline Panel & \multicolumn{10}{c}{ Number of ply orientations after adding layer } & Ply number in Minimum thickness \\
number & 0 & 15 & -15 & 30 & -30 & 45 & -45 & 60 & -60 & 75 & -75 & 90 & half laminate & in half laminate \\
\hline 1 & 1 & 0 & 0 & 4 & 4 & 2 & 2 & 1 & 1 & 0 & 0 & 2 & $17(+1)$ & 16 \\
2 & 0 & 0 & 0 & 3 & 3 & 2 & 2 & 1 & 1 & 0 & 0 & 2 & 14 & 14 \\
3 & 0 & 0 & 0 & 0 & 0 & 2 & 2 & 1 & 1 & 1 & 1 & 3 & $11(+1)$ & 10 \\
4 & 0 & 0 & 0 & 0 & 0 & 2 & 2 & 1 & 1 & 1 & 1 & 2 & $10(+1)$ & 9 \\
5 & 0 & 0 & 0 & 0 & 0 & 2 & 2 & 1 & 1 & 0 & 0 & 2 & 8 & 8 \\
6 & 0 & 0 & 0 & 0 & 0 & 2 & 2 & 1 & 1 & 1 & 1 & 3 & 11 & 11 \\
7 & 0 & 0 & 0 & 0 & 0 & 2 & 2 & 1 & 1 & 1 & 1 & 2 & $10(+1)$ & 9 \\
8 & 0 & 0 & 0 & 0 & 0 & 2 & 2 & 1 & 1 & 2 & 2 & 2 & 12 & 12 \\
9 & 1 & 0 & 0 & 5 & 5 & 2 & 2 & 1 & 1 & 0 & 0 & 2 & 19 & 19 \\
10 & 0 & 0 & 0 & 5 & 5 & 2 & 2 & 1 & 1 & 0 & 0 & 2 & $18(+1)$ & 17 \\
11 & 1 & 0 & 0 & 3 & 3 & 2 & 2 & 1 & 1 & 0 & 0 & 2 & 15 & 15 \\
12 & 0 & 0 & 0 & 3 & 3 & 2 & 2 & 1 & 1 & 0 & 0 & 2 & 14 & 14 \\
13 & 0 & 0 & 0 & 0 & 0 & 2 & 2 & 1 & 1 & 1 & 1 & 3 & 11 & 11 \\
14 & 0 & 0 & 0 & 0 & 0 & 2 & 2 & 1 & 1 & 0 & 0 & 3 & 9 & 9 \\
15 & 0 & 0 & 0 & 0 & 0 & 2 & 2 & 1 & 1 & 2 & 2 & 3 & $13(+1)$ & 12 \\
16 & 0 & 0 & 0 & 0 & 0 & 2 & 2 & 1 & 1 & 3 & 3 & 3 & 15 & 15 \\
17 & 0 & 0 & 0 & 0 & 0 & 2 & 2 & 1 & 1 & 1 & 1 & 2 & $10(+1)$ & 9 \\
18 & 0 & 0 & 0 & 0 & 0 & 2 & 2 & 1 & 1 & 1 & 1 & 3 & 11 & 11 \\
\hline
\end{tabular}

\begin{tabular}{|c|c|c|c|c|c|c|c|c|c|c|c|c|c|}
\hline \multirow{2}{*}{$\begin{array}{l}\text { Panel } \\
\text { number }\end{array}$} & \multicolumn{12}{|c|}{ Number of ply orientations after local blending } & \multirow{2}{*}{$\begin{array}{l}\text { Ply number in } \\
\text { half laminate }\end{array}$} \\
\hline & 0 & 15 & -15 & 30 & -30 & 45 & -45 & 60 & -60 & 75 & -75 & 90 & \\
\hline 1 & 1 & 0 & 0 & 4 & 4 & 2 & 2 & 1 & 1 & 0 & 0 & 2 & 17 \\
\hline 2 & 0 & 0 & 0 & 3 & 3 & 2 & 2 & 1 & 1 & 0 & 0 & 2 & 14 \\
\hline 3 & 0 & 0 & 0 & 1 & 1 & 2 & 2 & 1 & 1 & 0 & 0 & 3 & 11 \\
\hline 4 & 0 & 0 & 0 & 1 & 1 & 2 & 2 & 1 & 1 & 0 & 0 & 2 & 10 \\
\hline 5 & 0 & 0 & 0 & 0 & 0 & 2 & 2 & 1 & 1 & 0 & 0 & 2 & 8 \\
\hline 6 & 0 & 0 & 0 & 1 & 1 & 2 & 2 & 1 & 1 & 0 & 0 & 3 & 11 \\
\hline 7 & 0 & 0 & 0 & 1 & 1 & 2 & 2 & 1 & 1 & 0 & 0 & 2 & 10 \\
\hline 8 & 0 & 0 & 0 & 0 & 0 & 2 & 2 & 1 & 1 & 2 & 2 & 2 & 12 \\
\hline 9 & 1 & 0 & 0 & 5 & 5 & 2 & 2 & 1 & 1 & 0 & 0 & 2 & 19 \\
\hline 10 & 0 & 0 & 0 & 5 & 5 & 2 & 2 & 1 & 1 & 0 & 0 & 2 & 18 \\
\hline 11 & 1 & 0 & 0 & 3 & 3 & 2 & 2 & 1 & 1 & 0 & 0 & 2 & 15 \\
\hline 12 & 0 & 0 & 0 & 3 & 3 & 2 & 2 & 1 & 1 & 0 & 0 & 2 & 14 \\
\hline 13 & 0 & 0 & 0 & 1 & 1 & 2 & 2 & 1 & 1 & 0 & 0 & 3 & 11 \\
\hline 14 & 0 & 0 & 0 & 0 & 0 & 2 & 2 & 1 & 1 & 0 & 0 & 3 & 9 \\
\hline 15 & 0 & 0 & 0 & 1 & 1 & 2 & 2 & 1 & 1 & 1 & 1 & 3 & 13 \\
\hline 16 & 0 & 0 & 0 & 1 & 1 & 2 & 2 & 1 & 1 & 2 & 2 & 3 & 15 \\
\hline 17 & 0 & 0 & 0 & 1 & 1 & 2 & 2 & 1 & 1 & 0 & 0 & 2 & 10 \\
\hline 18 & 0 & 0 & 0 & 0 & 0 & 2 & 2 & 1 & 1 & 1 & 1 & 3 & 11 \\
\hline
\end{tabular}

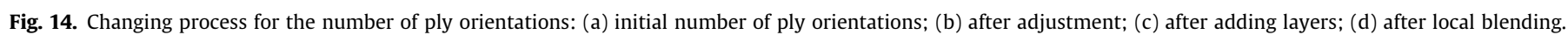




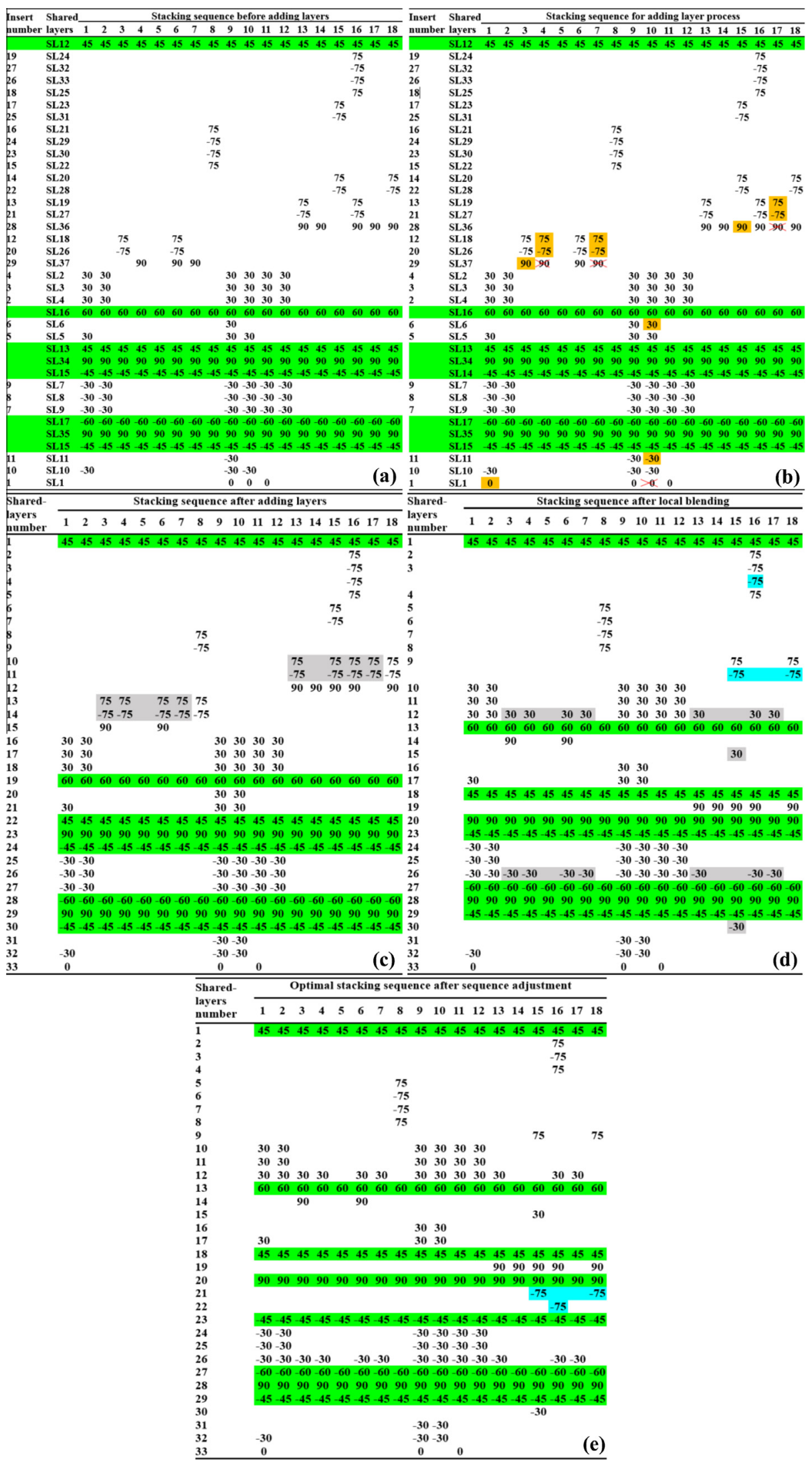

Fig. 15. Optimization process of stacking sequence: (a) initial stacking sequence; (b) adding layer process; (c) after adding layers; (d) after local blending; (e) Final optimal stacking sequence after sequence adjustment. 
Table 10

Results 2 of global shared layer blending method for the 18-panel problem.

\begin{tabular}{|c|c|c|c|c|c|c|c|c|c|c|c|c|c|c|c|c|c|c|c|}
\hline \multirow[t]{2}{*}{ Shared layers } & \multirow[t]{2}{*}{ Ply orientations } & \multicolumn{18}{|c|}{ Panels covered by shared layers } \\
\hline & & 1 & 2 & 3 & 4 & 5 & 6 & 7 & 8 & 9 & 10 & 11 & 12 & 13 & 14 & 15 & 16 & 17 & 18 \\
\hline SL1 & $0^{\circ}$ & & & & & & & & & 9 & 10 & 11 & & & & & & & \\
\hline & $15^{\circ}$ & & & & & & & & & & & & & & & & & & \\
\hline & $-15^{\circ}$ & & & & & & & & & & & & & & & & & & \\
\hline SL2 & $30^{\circ}$ & 1 & 2 & & & & & & & 9 & 10 & 11 & 12 & & & & & & \\
\hline SL3 & & 1 & 2 & & & & & & & 9 & 10 & 11 & 12 & & & & & & \\
\hline SL4 & & 1 & 2 & & & & & & & 9 & 10 & 11 & 12 & & & & & & \\
\hline SL5 & & 1 & & & & & & & & 9 & 10 & & & & & & & & \\
\hline SL6 & & & & & & & & & & 9 & & & & & & & & & \\
\hline SL7 & $-30^{\circ}$ & 1 & 2 & & & & & & & 9 & 10 & 11 & 12 & & & & & & \\
\hline SL8 & & 1 & 2 & & & & & & & 9 & 10 & 11 & 12 & & & & & & \\
\hline SL9 & & 1 & 2 & & & & & & & 9 & 10 & 11 & 12 & & & & & & \\
\hline SL10 & & 1 & & & & & & & & 9 & 10 & & & & & & & & \\
\hline SL11 & & & & & & & & & & 9 & & & & & & & & & \\
\hline SL12 & $45^{\circ}$ & 1 & 2 & 3 & 4 & 5 & 6 & 7 & 8 & 9 & 10 & 11 & 12 & 13 & 14 & 15 & 16 & 17 & 18 \\
\hline SL13 & & 1 & 2 & 3 & 4 & 5 & 6 & 7 & 8 & 9 & 10 & 11 & 12 & 13 & 14 & 15 & 16 & 17 & 18 \\
\hline SL14 & $-45^{\circ}$ & 1 & 2 & 3 & 4 & 5 & 6 & 7 & 8 & 9 & 10 & 11 & 12 & 13 & 14 & 15 & 16 & 17 & 18 \\
\hline SL15 & & 1 & 2 & 3 & 4 & 5 & 6 & 7 & 8 & 9 & 10 & 11 & 12 & 13 & 14 & 15 & 16 & 17 & 18 \\
\hline SL16 & $60^{\circ}$ & 1 & 2 & 3 & 4 & 5 & 6 & 7 & 8 & 9 & 10 & 11 & 12 & 13 & 14 & 15 & 16 & 17 & 18 \\
\hline SL17 & $-60^{\circ}$ & 1 & 2 & 3 & 4 & 5 & 6 & 7 & 8 & 9 & 10 & 11 & 12 & 13 & 14 & 15 & 16 & 17 & 18 \\
\hline SL18 & $75^{\circ}$ & & & 3 & & & 6 & & & & & & & & & & & & \\
\hline SL19 & & & & & & & & & & & & & & 13 & & & 16 & & \\
\hline SL20 & & & & & & & & & & & & & & & & 15 & & & 18 \\
\hline SL21 & & & & & & & & & 8 & & & & & & & & & & \\
\hline SL22 & & & & & & & & & 8 & & & & & & & & & & \\
\hline SL23 & & & & & & & & & & & & & & & & 15 & & & \\
\hline SL24 & & & & & & & & & & & & & & & & & 16 & & \\
\hline SL25 & & & & & & & & & & & & & & & & & 16 & & \\
\hline SL26 & $-75^{\circ}$ & & & 3 & & & 6 & & & & & & & & & & & & \\
\hline SL27 & & & & & & & & & & & & & & 13 & & & 16 & & \\
\hline SL28 & & & & & & & & & & & & & & & & 15 & & & 18 \\
\hline SL29 & & & & & & & & & 8 & & & & & & & & & & \\
\hline SL30 & & & & & & & & & 8 & & & & & & & & & & \\
\hline SL31 & & & & & & & & & & & & & & & & 15 & & & \\
\hline SL32 & & & & & & & & & & & & & & & & & 16 & & \\
\hline SL33 & & & & & & & & & & & & & & & & & 16 & & \\
\hline SL34 & $90^{\circ}$ & & 1 & 2 & 3 & 4 & 5 & 6 & 7 & 8 & 9 & 10 & 11 & 12 & 13 & 14 & 15 & 16 & 17 \\
\hline SL35 & & & 1 & 2 & 3 & 4 & 5 & 6 & 7 & 8 & 9 & 10 & 11 & 12 & 13 & 14 & 15 & 16 & 17 \\
\hline SL36 & & & & & & & & & & & & & & 13 & 14 & & 16 & 17 & 18 \\
\hline SL37 & & & & & 4 & & 6 & 7 & & & & & & & & & & & \\
\hline
\end{tabular}

Table 11

Variation of buckling load factors.

\begin{tabular}{|c|c|c|c|c|c|c|c|c|c|c|c|c|c|c|c|c|c|c|}
\hline \multirow{2}{*}{ Sequence - } & \multicolumn{18}{|c|}{ Buckling load factor for various panels } \\
\hline & 1 & 2 & 3 & 4 & 5 & 6 & 7 & 8 & 9 & 10 & 11 & 12 & 13 & 14 & 15 & 16 & 17 & 18 \\
\hline Fig. 15(a) & 0.9435 & 1.0064 & 0.9360 & 0.9774 & 1.1090 & 1.1314 & 0.9467 & 1.0000 & 1.0183 & 0.9268 & 1.0614 & 1.0021 & 1.1843 & 1.0367 & 0.9694 & 1.0670 & 0.9601 & $\overline{1.0261}$ \\
\hline Fig. 15(c) & 1.1272 & 1.0064 & 1.2589 & 1.3626 & 1.1090 & 1.1314 & 1.3199 & 1.0000 & 1.0183 & 1.1099 & 1.0614 & 1.0021 & 1.1843 & 1.0367 & 1.2399 & 1.0670 & 1.3386 & 1.0261 \\
\hline Fig. 15(e) & 1.1272 & 1.0064 & 1.1182 & 1.1925 & 1.1090 & 1.0049 & 1.1551 & 1.0000 & 1.0183 & 1.1099 & 1.0614 & 1.0021 & 1.0454 & 1.0258 & 1.1540 & 1.0074 & 1.1715 & 1.0132 \\
\hline
\end{tabular}

the continuity ply drops, the sequence-adjustment algorithm (Algorithm 9) is implemented. In Fig. 15(d) and (e), two subregion shared layers (highlighted with azure) are moved from the original stacking positions to the position between two global shared layers. The final optimal results are shown in Fig. 15(e) with corresponding layers in Fig. 16(c), (d) and sections in Fig. 17(b), (d). Compared to the sequence before local blending in Fig. 16 (a) and (b), the stacking position of global shared layers are changed in Fig. 16(c) since sequences are designed and adjusted and the subregion shared layers are fewer and bigger in Fig. 16(d). After local blending and sequence adjustment, as shown in Fig. 17, the continuity ply drops between panels $\{16,13\},\{17,14\},\{18,15\}$, $\{2,3\},\{2,6\},\{4,5\},\{12,13\},\{13,14\},\{14,15\},\{12,16\}$ and $\{16$, $17\}$ are decreased, especially for the layers between the panels with the ply orientation mismatch: $\{2,3\},\{2,6\},\{12,13\}$ and $\{12,16\}$. However, the continuity ply drops increased between panels $\{7,8\}$ and $\{17,18\}$ since the local blending improves the global blending property while ply drops are at the parts limited by the buckling constraint.

Buckling load factors of Fig. 15(c), (d) and (e) are presented in Table 11. The ply numbers and weight results for the optimal solution are shown in Table 12. The optimal results are compared with the previous studies, and the obtained weight is the smallest. Compared to the best result obtained by Fan [11], our solution is with an even number of plies in each panel and a total of 456 plies, 1 more ply than that of Fan. However, the solution of Fan has one more ply than ours in panels $1,2,9,10,11,12,16$ and 18 respectively, however each area of panels 1, 2, 9, 10, 11 and 12 is bigger than the other panels. In this work, the global shared layers are obtained by choosing preferable ply orientations with higher $\lambda_{c b}$ for larger area panels (Section 3.4). Layers are added to panels with small areas, as a result, the total weight is reduced. Most of the 

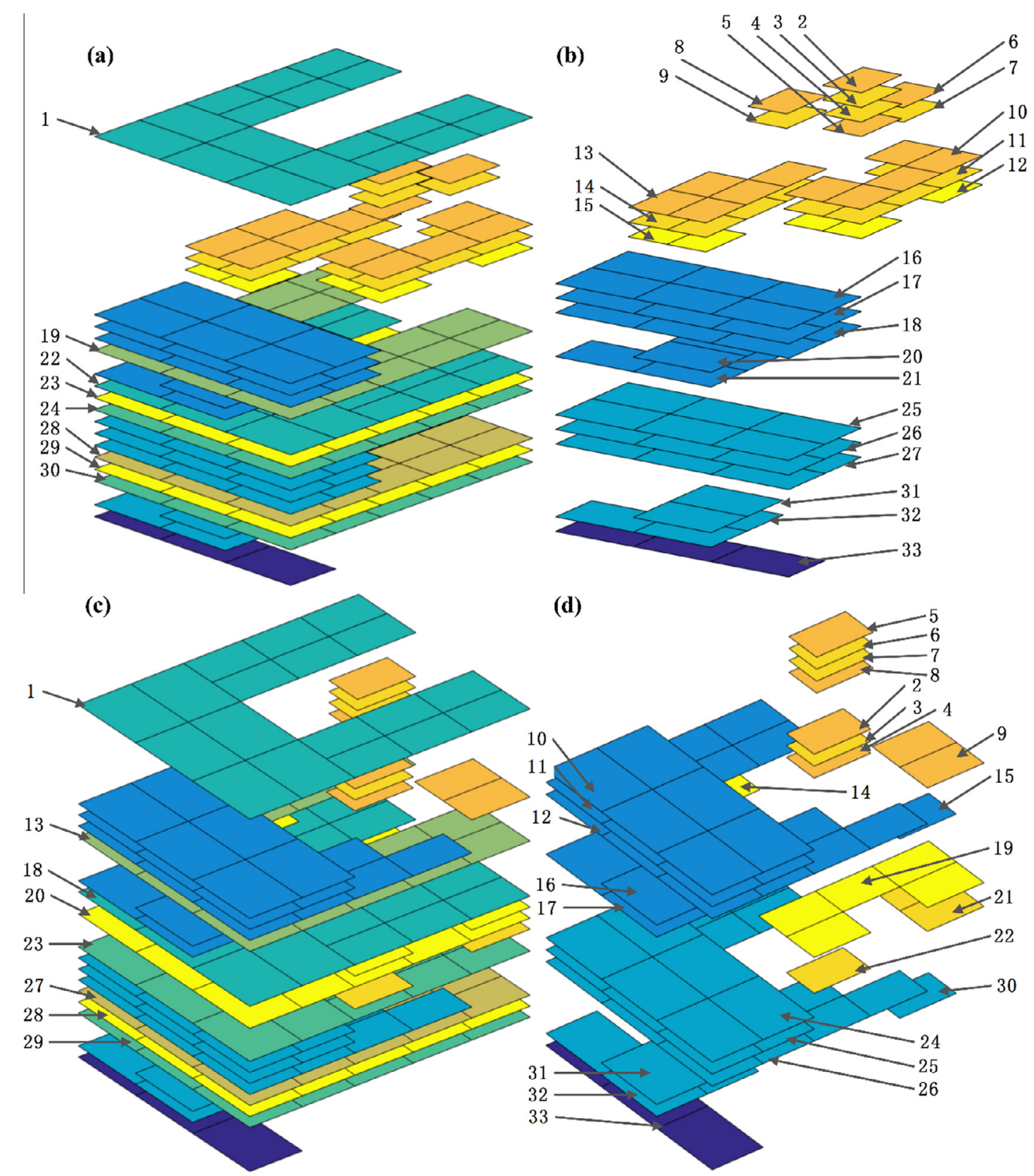

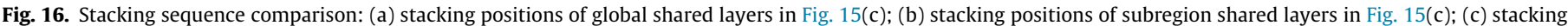
positions of global shared layers in Fig. 15(e); (d) stacking positions of subregion shared layers in Fig. 15(e).

solutions obtained by other researchers [4,6-11] cannot satisfy the constraints presented in Eq. (8) except that in [15] and the present solution.

The constraints presented in Eq. (8) should be checked: in Fig. 15(e) constraints CT1, CT2, CT3, CT4, CT5, CT6, CT11 and CT12 are satisfied, constraints $C T 7, C T 8, C T 9, C T 10$ and $C T 13$ can be checked with Fig. 17(b) and (d). The maximal number of continuity ply drops is 4 between panels $\{5,8\}$ and $\{7,8\}$, because there are 4 single layers in panel 8 limited by the buckling constraint. The $\Delta n-$ rule can be checked in Table 13 and Fig. 17(b), (d). Here, the ply drops in design are produced for two reasons:

(1) The thickness difference $\Delta h$ between the adjacent panels (also named natural ply drops).
(2) The ply-orientations mismatch $\Delta n-\Delta h$ between the adjacent panels.

Table 13 gives the number of ply drops between every two adjacent panels owing to different reasons. It is obviously that ply orientations mismatch often happens between adjacent panels with different best ply orientations in the MSPT. In panel 17, though it keeps maximal blending property with panel 16, poor blending quality appears with many ply drops between panels $\{14,17\}$ and $\{17,18\}$ due to buckling constraint for panels 14 and 18 . The maximal $\Delta h-\Delta n=8$ is between panels 12 and 16 (Fig. 17(d)); this should be improved in the subsequent design. However, all the constraints set in the objective function Eq. (8) are satisfied. A conclusion can be made from the above analysis: the smaller the ply orientations mismatch $\Delta n-\Delta h$ the better the blending 
(a)
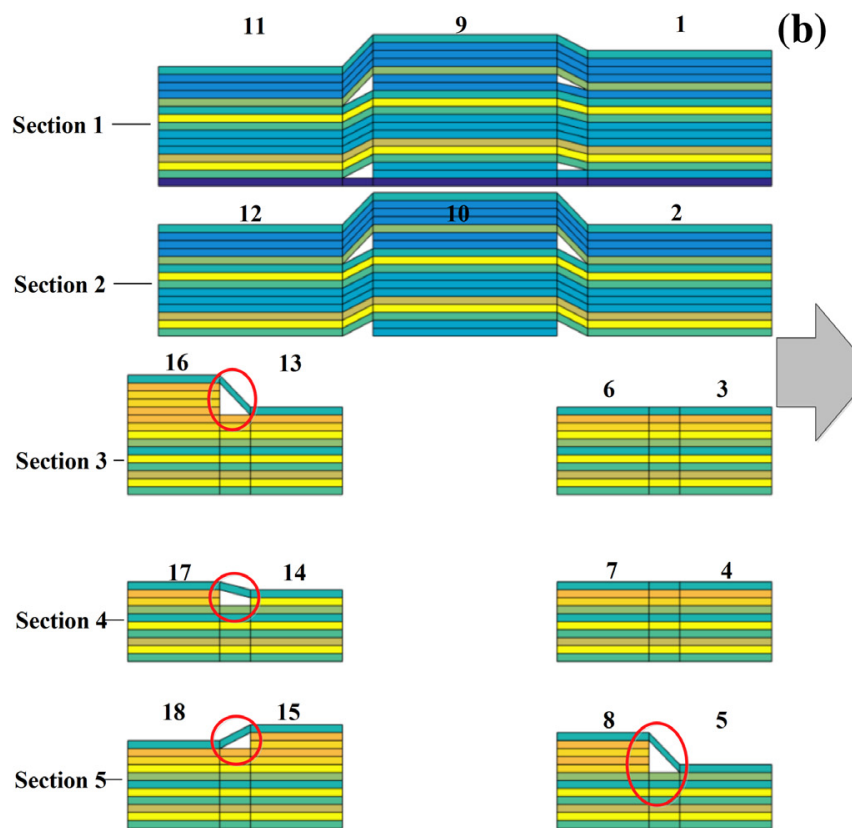

(c)

Section 6

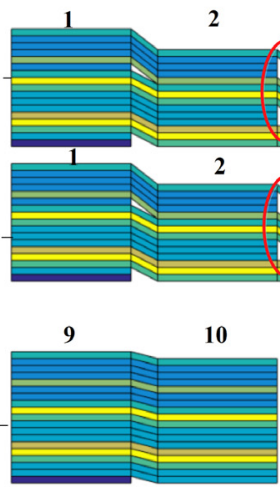

Section 8

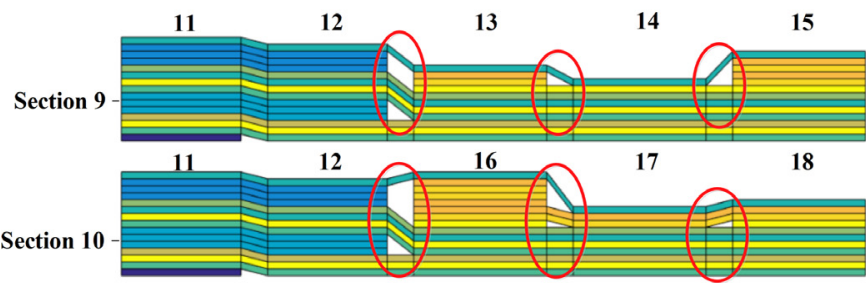

(d)

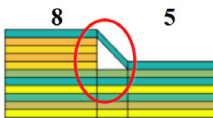

5
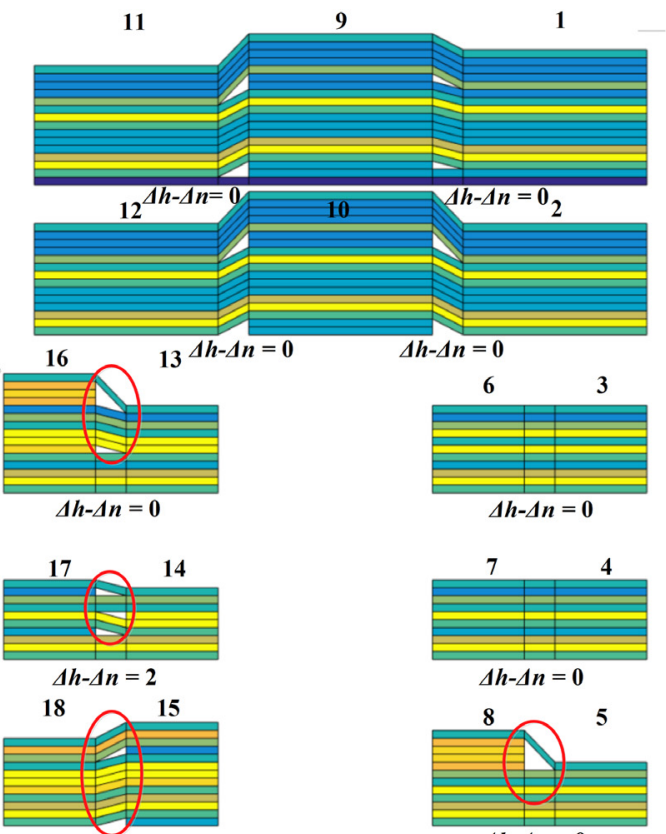

$\Delta h-\Delta n=0$

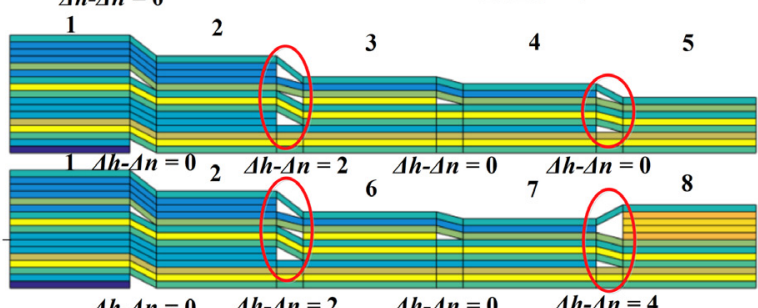

$\Delta h-\Delta n=0 \quad \Delta h-\Delta n=2 \quad \Delta h-\Delta n=0 \quad \Delta h-\Delta n=4$

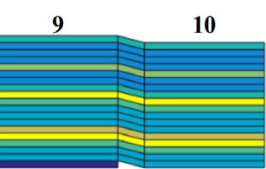

$\Delta h-\Delta n=0$

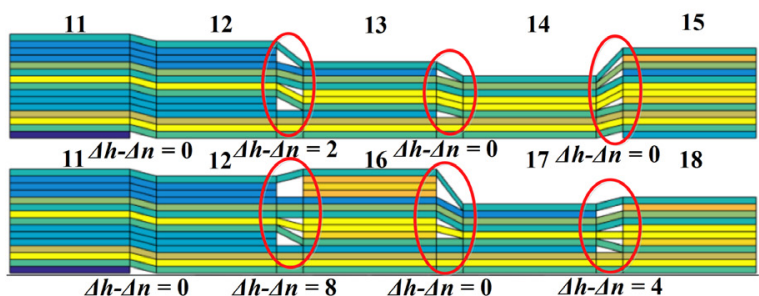

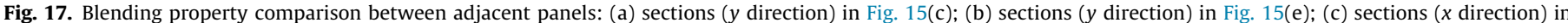
Fig. 15(c); (d) sections ( $x$ direction) in Fig. 15(e).

property; simultaneously, continuity ply drops should be minimized.

\section{Conclusions}

In this work, an entire framework for design and optimization of tapered composite structures is proposed, where the design concept extends shared layers from individual panels to the global blending structure. The optimization model for buckling analysis with multiple manufacturing constraints is formulated. The techniques used in the design process are developed from the classical lamination theory (e.g. MSPT etc.). Due to the complex of high-dimensional discrete variables and different types of constraints in individual panel and the whole structure, the variables are divided and dealt with at various levels, and the constraints were enforced at different steps to increase the design flexibility and improve the computational efficiency.

An 18-panel benchmark problem is adopted to validate the proposed framework; the obtained results were compared with those of previous studies. Owing to the high-dimensional variable space, there are many local optima. The efficiency of this framework implies its potential for design and optimization of large-scale composite structures. However, the strategies for choosing ply orientations, improving blending properties and sequence optimization can be further improved in future work. 


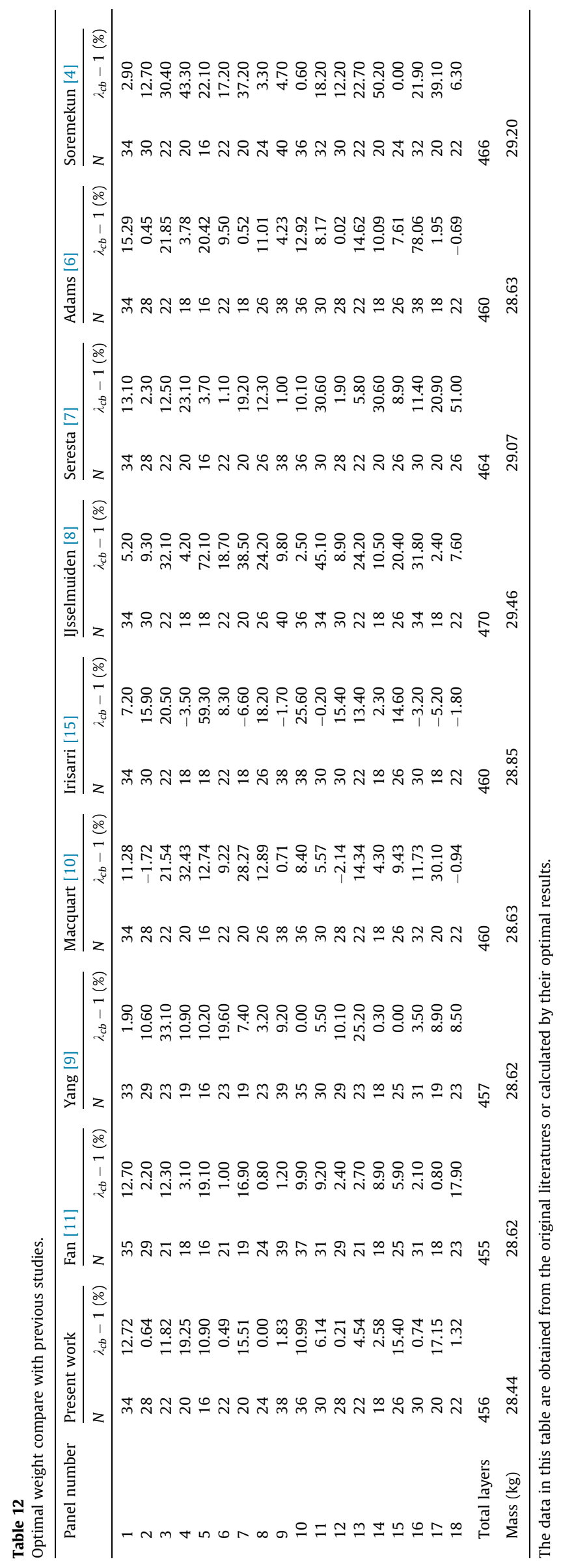

Table 13

Ply drops analysis of the optimal solution in Fig. 15(e).

\begin{tabular}{|c|c|c|c|}
\hline $\begin{array}{l}\text { Adjacent } \\
\text { panels }\end{array}$ & $\begin{array}{l}\text { Thickness difference } \\
\Delta h\end{array}$ & $\Delta n$ & $\begin{array}{l}\text { Ply orientation mismatch } \\
\Delta n-\Delta h\end{array}$ \\
\hline$\{1,2\}$ & 3 & 3 & 0 \\
\hline$\{1,9\}$ & 2 & 2 & 0 \\
\hline$\{2,3\}$ & 3 & 5 & 2 \\
\hline$\{2,6\}$ & 3 & 5 & 2 \\
\hline$\{2,10\}$ & 4 & 4 & 0 \\
\hline$\{3,4\}$ & 1 & 1 & 0 \\
\hline$\{3,6\}$ & 0 & 0 & 0 \\
\hline$\{4,5\}$ & 2 & 2 & 0 \\
\hline$\{4,7\}$ & 0 & 0 & 0 \\
\hline$\{5,8\}$ & 4 & 4 & 0 \\
\hline$\{6,7\}$ & 1 & 1 & 0 \\
\hline$\{7,8\}$ & 2 & 6 & 4 \\
\hline$\{9,10\}$ & 1 & 1 & 0 \\
\hline$\{9,11\}$ & 4 & 4 & 0 \\
\hline$\{10,12\}$ & 4 & 4 & 0 \\
\hline$\{11,12\}$ & 1 & 1 & 0 \\
\hline$\{12,13\}$ & 3 & 5 & 2 \\
\hline$\{12,16\}$ & 1 & 9 & 8 \\
\hline$\{13,14\}$ & 2 & 2 & 0 \\
\hline$\{13,16\}$ & 4 & 4 & 0 \\
\hline$\{14,15\}$ & 4 & 4 & 0 \\
\hline$\{14,17\}$ & 1 & 3 & 2 \\
\hline$\{15,18\}$ & 2 & 2 & 0 \\
\hline$\{16,17\}$ & 5 & 5 & 0 \\
\hline$\{17,18\}$ & 1 & 5 & 4 \\
\hline Total & 58 & 82 & 24 \\
\hline
\end{tabular}

\section{Acknowledgement}

This work is supported by the National Natural Science Foundation of China (No. 51375386) and China Scholarship Council (CSC).

\section{References}

[1] He K, Hoa SV, Ganesan R. The study of tapered laminated composite structures: a review. Compos Sci Technol 2000;60:2643-57.

[2] Kristinsdottir BP, Zabinsky ZB, Tuttle ME, Neogi S. Optimal design of large composite panels with varying loads. Compos Struct 2001;51(1):93-102.

[3] Ghiasi H, Pasini D, Lessard L. Optimum stacking sequence design of composite materials Part I: constant stiffness design. Compos Struct 2009;90:1-11.

[4] Soremekun G, Gürdal Z, Kassapoglou C, Toni D. Stacking sequence blending of multiple composite laminates using genetic algorithms. Compos Struct 2002;56(1):53-62.

[5] Adams DB, Watson LT, Gürdal Z. Optimization and blending of composite laminates using genetic algorithms with migration. Mech Adv Mater Struct 2003;10(3):183-203.

[6] Adams DB, Watson LT, Gürdal Z, Anderson-Cook CM. Genetic algorithm optimization and blending of composite laminates by locally reducing laminate thickness. Adv Eng Softw 2004;35(1):35-43.

[7] Seresta O, Abdalla MM, Gürdal Z. A genetic algorithm based blending scheme for design of multiple composite laminates. In: Proceedings of 50th AIAA/ ASME/ASCE/AHS/ASC structures, structural dynamics, and materials conference, Palm Springs, California, USA

[8] IJsselmuiden ST, Abdalla MM, Seresta O, Gürdal Z. Multi-step blended stacking sequence design of panel assemblies with buckling constraints. Compos B Eng 2009;40:329-36.

[9] Yang JB, Song BF, Zhong XP, Jin P. Optimal design of blended composite laminate structures using ply drop sequence. Compos Struct 2016;135:30-7.

[10] Macquart T, Bordogna MT, Lancelot P, Breuker RD. Derivation and application of blending constraints in lamination parameter space for composite optimisation. Compos Struct 2016;135:224-35.

[11] Fan H-T, Wang H, Chen X-H. An optimization method for composite structures with ply-drops. Compos Struct 2016;136:650-61.

[12] Seresta O, Gürdal Z, Adams DB, Watson LT. Optimal design of composite wing structures with blended laminates. In: 10th AIAA/ISSMO multidisciplinary analysis and optimization conference. p. 1-13.

[13] Adams DB, Watson LT, Setoodeh O, Gürdal Z. Global/local iteration for blended composite laminate panel structure optimization subproblems. Mech Adv Mater Struct 2007;14(2):139-50.

[14] Liu DZ, Toropov VV, Querin OM, Barton DC. Bi-level optimization of blended composite panels. In: 50th AIAA/ASME/ASCE/AHS/ASC structures, structural dynamics, and materials conference 17th, Palm Springs, California.

[15] Irisarri F-X, Lasseigne A, Leroy FH, Riche RL. Optimal design of laminated composite structures with ply drops using stacking sequence tables. Compos Struct 2014;17:559-69. 
[16] Zein S, Colson B, Grihon S. A primal-dual backtracking optimization method for blended composite structures. Struct Multidiscip Optim 2012;45:669-80.

[17] Zein S, Basso P, Grihon S. A constraint satisfaction programming approach for computing manufacturable stacking sequences. Comput Struct 2014;136:56-63.

[18] Zein S, Bruyneel M. A bilevel integer programming method for blended composite structures. Adv Eng Softw 2015;79:1-12.

[19] Panesar AS, Weaver PM. Optimisation of blended bistable laminates for a morphing flap. Compos Struct 2012:94:3092-105.

[20] Lund E. Buckling topology optimization of laminated multi-material composite shell structures. Compos Struct 2009;91:158-67.

[21] Bruyneel M, Beghin C, Craveur G, Grihon S, Sosonkina M. Stacking sequence optimization for constant stiffness laminates based on a continuous optimization approach. Struct Multidiscip Optim 2012;46:783-94.

[22] Zhou M, Fleury R, Kemp M. Optimization of composite: recent advances and application. In: The 7th altair CAE technology conference, Altair.

[23] Jing Z, Fan XL, Sun Q. Stacking sequence optimization of composite laminates for maximum buckling load using permutation search algorithm. Compos Struct 2015;121:225-36.
[24] Jing Z, Fan XL, Sun Q. Global shared-layer blending method for stacking sequence optimization design and blending of composite structures. Compos B Eng 2015;69:181-90.

[25] Jing Z, Sun Q Silberschmidt VV. Sequential permutation table method for optimization of stacking sequence in composite laminates. Compos Struct 2016.

[26] Bloomfield MW, Diaconu CG, Weaver PM. On feasible regions of lamination parameters for lay-up optimization of laminated composites. Proc R Soc A 2009;465:1123-43.

[27] Marmaras K. Optimal design of composite structures under manufacturing constraints [Ph.D. thesis]. Aalborg University; 2014.

[28] Dillinger JKS. Static aeroelastic optimization of composite wings with variable stiffness laminates [Ph.D. thesis]. Delft University of Technology; 2015.

[29] Leissa AW. Buckling of laminated composite plates and shell panels, Repor AFWAL-TR-85-3069. AF Wright Aeronautical Laboratories; 1985.

[30] Tsai SW, Hahn HT. Introduction to composite materials. Westport: Technomic; 1980. 